Supporting Information

\title{
[3+2] Cross-Coupling Reactions of Aziridines with Isocyanates Catalyzed by Nickel(II) Iodide
}

Takeshi Munegumi, ${ }^{\dagger}$ Isao Azumaya, ${ }^{\ddagger}$ Takako Kato, ${ }^{\ddagger}$ Hyuma Masu, ${ }^{\ddagger}$ Shinichi Saito ${ }^{*},{ }^{\dagger}$

Department of Chemistry, Faculty of Science, Tokyo University of Science, Kagurazaka, Shinjuku, Tokyo, Japan 162-8601, and Faculty of Pharmaceutical Sciences at Kagawa Campus, Tokushima Bunri University, Kagawa, Japan 769-2193

${ }^{\dagger}$ Tokyo University of Science ${ }^{\ddagger}$ Tokushima Bunri University 


\section{Analytical Methods}

${ }^{1} \mathrm{H}$ NMR spectra were recorded on a JEOL JNM-EX270L FT NMR SYSTEM (270 MHz) , a JEOL JNM-AL300 FT NMR SYSTEM (300 MHz), or a Bruker AM-600 (600 MHz). Chemical Shifts were reported in delta unit ( $\delta$ relative to chloroform- $d$ (7.24 ppm for ${ }^{1} \mathrm{H}$ NMR and $77.0 \mathrm{ppm}$ for ${ }^{13} \mathrm{C}$ NMR)). Multiplicity is indicated by s (single), d (doublet), t (triplet), and m (multiplet). Coupling constants, $J$, are reported in Hz. IR spectra were recorded on a JASCO FT/IR-410 (FT-IR). Elemental analyses were measured by YANACO CHN-recorder MT-6. Thin layer chromatography (TLC) was performed using Merck silica gel 60F-254 plates.

\section{General procedure}

Aziridines $\mathbf{1} \mathbf{a}^{\mathbf{1}}, \mathbf{1 b}-\mathbf{c},{ }^{2} \mathbf{1 e},{ }^{3,4} \mathbf{1 f},{ }^{3} \mathbf{1 g},{ }^{1}$ and $\mathbf{1}{ }^{5}$ were prepared as reported in the literature. Aziridine $\mathbf{1 d}^{\mathbf{6}}$ was prepared from 2-aziridin-1-yl-ethanol (commercially available). Isocyanates 2a-e were commercially available and used without further purification. Column chromatography was performed with silica gel 60N (spherical, neutral, 63-210 mm, purchased from Kanto Kagaku Co., LTD).

1a

${ }^{1} \mathrm{H}$ NMR (600 MHz, CDCl $)$ 7.27-7.17 (m, 5H), 3.30 (s, 2H), 1.74-1.73 (m, 2H), 1.20-1.19 (m, 2H);

${ }^{13} \mathrm{C}$ NMR (150 MHz, $\mathrm{CDCl}_{3}$ ) 139.3, 128.3, 128.0, 127.0, 65.3, 27.5.

$1 \mathbf{b}$

${ }^{1} \mathrm{H}$ NMR (600 MHz, CDCl $) 2.15$ (t, $\left.J=7.2 \mathrm{~Hz}, 2 \mathrm{H}\right), 1.68-1.67$ (m, 2H), 1.55-1.50 (m, 2H), 1.38-1.35 (m, 2H), 1.04-1.03 (m, 2H), 0.90 (t, $J=7.8 \mathrm{~Hz}, 3 \mathrm{H}) ;{ }^{13} \mathrm{C}$ NMR (150 MHz, $\left.\mathrm{CDCl}_{3}\right) 61.8$, 32.0, 27.1, 20.5, 14.0 .

1c

${ }^{1} \mathrm{H}$ NMR (300 MHz, $\mathrm{CDCl}_{3}$ ) 1.46-1.45 (m, 2H), 1.35-1.33 (m, 2H), 0.93 (s, 9H); ${ }^{13} \mathrm{C}$ NMR (150 $\left.\mathrm{MHz}, \mathrm{CDCl}_{3}\right)$ 52.5, 26.2, 20.1.

$1 \mathrm{~g}$

${ }^{1} \mathrm{H}$ NMR (300 MHz, $\mathrm{CDCl}_{3}$ ) 7.27-7.22 (m, 2H), 7.04-6.94 (m, 3H), 2.11 (s, 4H); ${ }^{13} \mathrm{C}$ NMR (150 $\left.\mathrm{MHz}, \mathrm{CDCl}_{3}\right)$ 155.1, 128.9, 122.3, 121.0, 27.5.

\section{Preparation of aziridine 1d}

To a mixture of 2-aziridin-1-yl-ethanol (1.0 ml, $12.5 \mathrm{mmol})$ in THF (20 ml) was slowly added $\mathrm{NaH}$ $(0.75 \mathrm{~g}, 18.7 \mathrm{mmol})$ at $0{ }^{\circ} \mathrm{C}$. Then, TBSCl $(2.8 \mathrm{~g}, 18.7 \mathrm{mmol})$ was slowly added. When the exothermic reaction had subsided, the resulting solution allowed to warm to r.t, and stirred for $3 \mathrm{~h}$. Then, the mixture was quenched with water, extracted with dichloromethane, and the combined organic phases were dried over sodium sulfate. Evaporation of the solvent gave oil, which was further purified by silica gel column chromatography $\left(\mathrm{CH}_{2} \mathrm{Cl}_{2} / \mathrm{MeOH}, 30: 1\right)$ to yield $\mathbf{1 d}(1.4 \mathrm{~g}, 7.0$ 
mmol, $56 \%)$.

yellow oil; ${ }^{1} \mathrm{H}$ NMR (600 MHz, $\left.\mathrm{CDCl}_{3}\right) 3.79$ (t, $\left.J=6.0 \mathrm{~Hz}, 2 \mathrm{H}\right), 2.34$ (t, $\left.J=6.0 \mathrm{~Hz}, 2 \mathrm{H}\right), 1.72-1.71$

(m, 2H), 1.17-1.16 (m, 2H), 0.90 (s, 9H), 0.07 (s, 6H); ${ }^{13} \mathrm{C} \mathrm{NMR} \mathrm{(150} \mathrm{MHz,} \mathrm{CDCl}_{3}$ ) 63.6, 63.0, 26.9, 25.9, 18.3, -5.4 .

\section{Nickel catalyzed cross coupling aziridines 1 with isocyanates 2}

A Representative Procedure. To a purple mixture of $\mathrm{NiI}_{2}(0.0312 \mathrm{~g}, 0.1 \mathrm{mmol})$ in dry toluene (3.5 $\mathrm{mL}$ ) was added a solution of $\mathbf{1}(1 \mathrm{mmol})$ in dry toluene $(3.5 \mathrm{~mL})$ under an argon atmosphere at r.t. Then, compound 2 ( $1.5 \mathrm{mmol}$ ) was added, and the mixture was stirred at $100{ }^{\circ} \mathrm{C}$. The progress of the reaction was monitored by TLC and/or GC-MS, and the mixture was stirred until the starting material 1 disappeared. The mixture was passed through a short column of alumina $\left(\mathrm{CH}_{2} \mathrm{Cl}_{2}\right)$. Evaporation of the solvent gave oil, which was further purified by silica gel column chromatography (hexane / AcOEt) to give $\mathbf{3}$ or $\mathbf{4}$.

\section{Nickel catalyzed isomerization of 3}

To a purple mixture of $\mathrm{NiI}_{2}(0.0312 \mathrm{~g}, 0.1 \mathrm{mmol})$ in dry toluene $(3.5 \mathrm{~mL})$ was added a solution of 3fa $(0.294 \mathrm{~g}, 1 \mathrm{mmol})$ in dry toluene $(3.5 \mathrm{~mL})$ under an argon atmosphere at r.t., and the mixture was stirred for $16 \mathrm{~h}$ at $100{ }^{\circ} \mathrm{C}$. The mixture was passed through a short column of alumina $\left(\mathrm{CH}_{2} \mathrm{Cl}_{2}\right)$. Evaporation of the solvent gave oil, which was further purified by silica gel column chromatography (hexane / AcOEt) to give $\mathbf{4 f a}$.

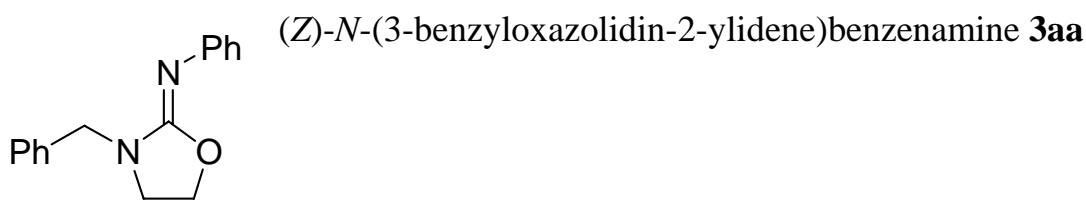

white solid, mp 98-99 ${ }^{\circ} \mathrm{C}$; ${ }^{1} \mathrm{H}$ NMR (300 MHz, $\mathrm{CDCl}_{3}$ ) 7.39-6.93 (m, 10H), 4.57 (s, 2H), 4.29 (t, $J=$ $7.2 \mathrm{~Hz}, 2 \mathrm{H}), 3.36$ (t, $J=7.5 \mathrm{~Hz}, 2 \mathrm{H}) ;{ }^{13} \mathrm{C}$ NMR $\left(75 \mathrm{MHz}, \mathrm{CDCl}_{3}\right)$ 153.2, 147.8, 136.7, 128.6, 128.5, 128.3, 127.6, 123.4, 122.0, 64.4, 49.2, 45.1; IR (KBr) 3426, 2856, 2370, 1673, 1592, 1488, 1448 , 1355, 1263, $1170 \mathrm{~cm}^{-1}$; Anal. Calcd for $\mathrm{C}_{16} \mathrm{H}_{16} \mathrm{~N}_{2} \mathrm{O}$ : C, 76.16; H, 6.39; N, 11.10. Found: C, 75.92; H, 6.44; N, 10.94 . 
<smiles>O=C1N(Cc2ccccc2)CCN1c1ccccc1</smiles>

white solid, mp 117-118 ${ }^{\circ} \mathrm{C} ;{ }^{1} \mathrm{H}$ NMR (300 MHz, $\left.\mathrm{CDCl}_{3}\right) 7.52$ (d, $\left.J=8.1 \mathrm{~Hz}, 2 \mathrm{H}\right), 7.31-7.18$ (m, 7H), 6.97 (t, $J=7.5 \mathrm{~Hz}, 1 \mathrm{H}$ ), 4.41 (s, 2H), 3.71 (dd, $J=7.8,9.9 \mathrm{~Hz}, 2 \mathrm{H}$ ), 3.28 (dd, $J=6.0,8.1 \mathrm{~Hz}$, 2H); ${ }^{13} \mathrm{C}$ NMR (75 MHz, $\mathrm{CDCl}_{3}$ ) 157.7, 140.5, 136.8, 128.8, 128.6, 128.2, 127.5, 122.3, 117.3, 48.1, 42.3, 41.2; IR (KBr) 3060, 3029, 2898, 1689, 1596, 1484, 1428, 1361, 1268, 1209, 1155, $1089 \mathrm{~cm}^{-1}$; Anal. Calcd for $\mathrm{C}_{16} \mathrm{H}_{16} \mathrm{~N}_{2} \mathrm{O}$ : C, 76.16; H, 6.39; N, 11.10. Found: C, 75.96; H, 6.50; N, 11.06.

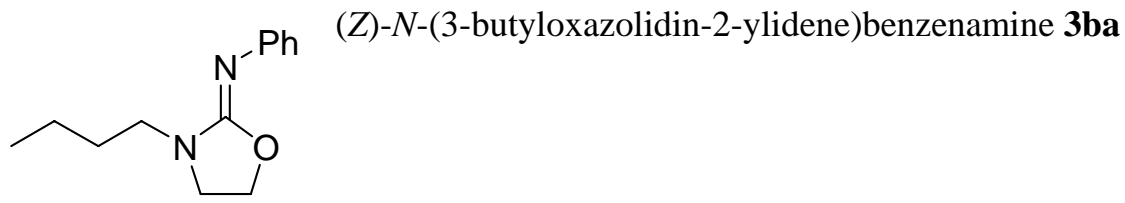

yellow oil; ${ }^{1} \mathrm{H}$ NMR $\left(600 \mathrm{MHz}, \mathrm{CDCl}_{3}\right) 7.21(\mathrm{t}, J=7.8 \mathrm{~Hz}, 2 \mathrm{H}), 7.01(\mathrm{~d}, J=7.8 \mathrm{~Hz} 1 \mathrm{H}), 693(\mathrm{t}, J=$ $7.8 \mathrm{~Hz}, 2 \mathrm{H}), 4.28$ (t, $J=7.2 \mathrm{~Hz}, 2 \mathrm{H}), 3.48$ (t, $J=7.8 \mathrm{~Hz}, 2 \mathrm{H}), 3.36$ (t, $J=7.8 \mathrm{~Hz}, 2 \mathrm{H}), 1.62-1.57(\mathrm{~m}$, 2H), 1.42-1.36 (m, 2H), 0.96 (t, $J=7.2 \mathrm{~Hz}, 3 \mathrm{H}) ;{ }^{13} \mathrm{C}$ NMR (150 MHz, $\left.\mathrm{CDCl}_{3}\right)$ 153.3, 148.1, 128.4, 123.4, 121.8, 64.3, 45.7, 44.9, 29.4, 20.0, 13.9; IR (neat) 2959, 2929, 2870, 1675, 1590, 1479, 1429, 1366, 1261, 1195, 1117, $1028 \mathrm{~cm}^{-1}$; HRMS Calcd for $\mathrm{C}_{13} \mathrm{H}_{18} \mathrm{~N}_{2} \mathrm{O}$ : 218.1419. Found: 218.1410.

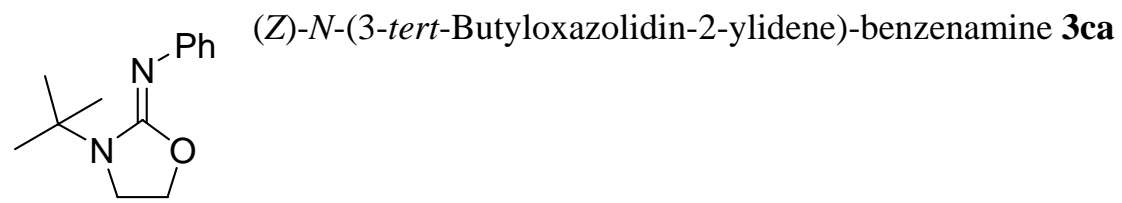

yellow oil; ${ }^{1} \mathrm{H}$ NMR (600 MHz, $\left.\mathrm{CDCl}_{3}\right) 7.22$ (t, J= 7.8 Hz, 2H), 7.00 (d, $\left.J=7.8 \mathrm{~Hz}, 2 \mathrm{H}\right), 6.93(\mathrm{t}, J=$ $7.2 \mathrm{~Hz}, 1 \mathrm{H}), 4.15$ (t, $J=7.2 \mathrm{~Hz}, 2 \mathrm{H}), 3.50$ (t, $J=7.8 \mathrm{~Hz}, 2 \mathrm{H}), 1.49$ (s, 9H); ${ }^{13} \mathrm{C}$ NMR (150 MHz, $\mathrm{CDCl}_{3}$ ) 151.1, 148.5, 128.4, 123.2, 121.5, 63.4, 53.6, 44.4, 27.0; IR (neat) 2973, 2907, 1683, 1590, 1478, 1398, 1360, 1293, 1245, 1141, 1049, $980 \mathrm{~cm}^{-1}$; HRMS Calcd for $\mathrm{C}_{13} \mathrm{H}_{18} \mathrm{~N}_{2} \mathrm{O}$ : 218.1419. Found: 218.1421. 


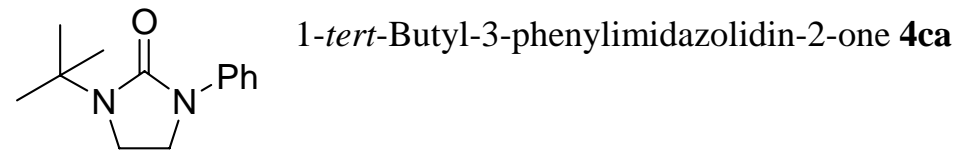

white solid, mp 90-91 ${ }^{\circ} \mathrm{C}$; ${ }^{1} \mathrm{H}$ NMR (270 MHz, $\mathrm{CDCl}_{3}$ ) 7.52-7.49 (m, 2H), 7.32-7.26 (m, 2H), 7.02-6.96 (m, 1H), 3.68 (dt, $J=1.0,7.5 \mathrm{~Hz}, 2 \mathrm{H}), 3.47$ (dt, $J=1.0,7.3 \mathrm{~Hz}, 2 \mathrm{H}), 1.40(\mathrm{~s}, 9 \mathrm{H}) ;{ }^{13} \mathrm{C}$ NMR (150 MHz, $\mathrm{CDCl}_{3}$ ) 158.0, 140.7, 128.7, 122.2, 117.6, 53.5, 42.1, 40.0, 27.5; IR (KBr) 2980, 2954, 2865, 1686, 1597, 1506, 1455, 1400, 1361, 1323, 1249, 1156, 1020, $895 \mathrm{~cm}^{-1}$; Anal. Calcd for $\mathrm{C}_{13} \mathrm{H}_{18} \mathrm{~N}_{2} \mathrm{O}$ : C, 71.53; H, 8.31; N, 12.83. Found: C, 71.28; H, 8.33; N, 12.62.

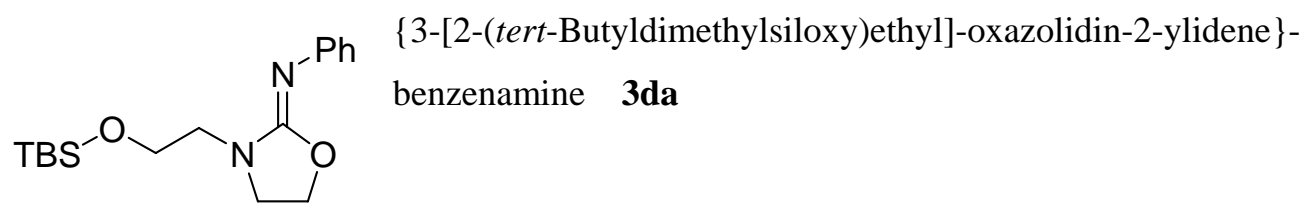

yellow oil; ${ }^{1} \mathrm{H}$ NMR (600 MHz, $\left.\mathrm{CDCl}_{3}\right) 7.21$ (t, $J=8.4 \mathrm{~Hz}, 2 \mathrm{H}$ ), 7.00 (d, $J=7.2 \mathrm{~Hz}, 2 \mathrm{H}$ ), 6.93 (t, $J$ $=7.2 \mathrm{~Hz}, 1 \mathrm{H}), 4.28(\mathrm{t}, J=7.8 \mathrm{~Hz}, 2 \mathrm{H}), 3.86(\mathrm{t}, J=5.4 \mathrm{~Hz}, 2 \mathrm{H}), 3.67$ (t, $J=7.8 \mathrm{~Hz}, 2 \mathrm{H}), 3.49$ (t, $J=$ $4.8 \mathrm{~Hz}, 2 \mathrm{H}), 0.89$ (s, 9H), 0.07 (s, 6H); ${ }^{13} \mathrm{C}$ NMR (75 MHz, $\mathrm{CDCl}_{3}$ ) 153.0, 148.0, 129.0, 128.9, 128.4, 128.2, 123.4, 121.9, 64.6, 61.9, 47.7, 47.7, 25.9, 18.2, -5.4; IR (neat) 3056, 2954, 2928, 2884, 2857, 1681, 1591, 1544, 1478, 1361, 1256, 1196, $1104 \mathrm{~cm}^{-1}$; HRMS Calcd for $\mathrm{C}_{17} \mathrm{H}_{28} \mathrm{~N}_{2} \mathrm{O}_{2} \mathrm{Si}$ : 320.1920. Found: 320.1919.

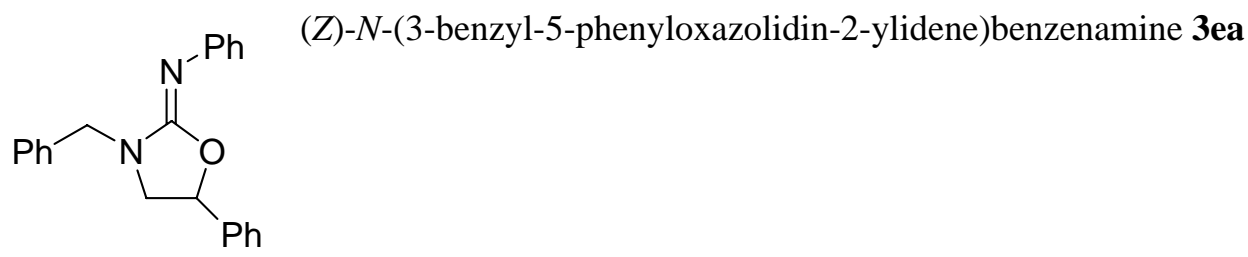

white solid, mp 88-90 ${ }^{\circ} \mathrm{C} ;{ }^{1} \mathrm{H}$ NMR $\left(300 \mathrm{MHz}, \mathrm{CDCl}_{3}\right.$ ) 7.39-7.16 (m, 14H), 6.95 (tt, $J=7.2,1.2 \mathrm{~Hz}$, 1H), 5.45 (t, $J=7.8 \mathrm{~Hz}, 1 \mathrm{H}$ ), 4.72 (d, $J=14.7 \mathrm{~Hz}, 1 \mathrm{H}$ ), 4.53 (d, $J=14.7 \mathrm{~Hz}, 1 \mathrm{H}), 3.71$ (t, $J=8.4 \mathrm{~Hz}$, $1 \mathrm{H}), 3.26$ (dd, $J=8.7,7.8 \mathrm{~Hz}, 1 \mathrm{H}) ;{ }^{13} \mathrm{C} \mathrm{NMR}\left(75 \mathrm{MHz}, \mathrm{CDCl}_{3}\right)$ 152.7, 147.4, 138.6, 136.6, 128.7, 128.7, 128.7, 128.5, 128.3, 127.6, 125.8, 123.6, 122.0, 77.4, 52.6, 49.3; IR (KBr) 3030, 2865, 2361, 1681, 1590, 1496, 1477, 1236, $1020 \mathrm{~cm}^{-1}$; Anal. Calcd for $\mathrm{C}_{22} \mathrm{H}_{20} \mathrm{~N}_{2} \mathrm{O}$ : C, 80.46; H, 6.14; N, 8.53. Found: C, 80.37; H, 6.24; N, 8.44 . 
<smiles>O=C1N(Cc2ccccc2)CC(c2ccccc2)N1c1ccccc1</smiles>

white solid, mp 134-136 ${ }^{\circ} \mathrm{C}$; ${ }^{1} \mathrm{H}$ NMR $\left(300 \mathrm{MHz}, \mathrm{CDCl}_{3}\right) 7.37$ (d, $\left.J=7.8 \mathrm{~Hz}, 2 \mathrm{H}\right)$, 7.22-7.11 (m, 13H), 6.89 (t, $J=7.5 \mathrm{~Hz}, 1 \mathrm{H}), 4.37$ (s, $2 \mathrm{H}), 4.54$ (d, $J=14.7 \mathrm{~Hz}, 1 \mathrm{H}), 4.33$ (d, $J=14.7 \mathrm{~Hz}, 1 \mathrm{H})$, $3.66(\mathrm{t}, J=9.0 \mathrm{~Hz}, 1 \mathrm{H}), 3.03(\mathrm{dd}, J=9.0,5.7 \mathrm{~Hz}, 1 \mathrm{H}) ;{ }^{13} \mathrm{C} \mathrm{NMR}\left(75 \mathrm{MHz}, \mathrm{CDCl}_{3}\right) 158.2,140.4$, 139.2, 136.7, 129.0, 128.6, 128.5, 128.2, 128.0, 127.6, 126.0, 122.9, 119.8, 57.6, 50.9, 48.0; IR (KBr) 3061, 3038, 1691, 1598, 1503, 1441, 1352, 1256, $1142 \mathrm{~cm}^{-1}$; Anal. Calcd for $\mathrm{C}_{22} \mathrm{H}_{20} \mathrm{~N}_{2} \mathrm{O}$ : C, 80.46; H, 6.14; N, 8.53. Found: C, 80.76; H, 6.23; N, 8.37.

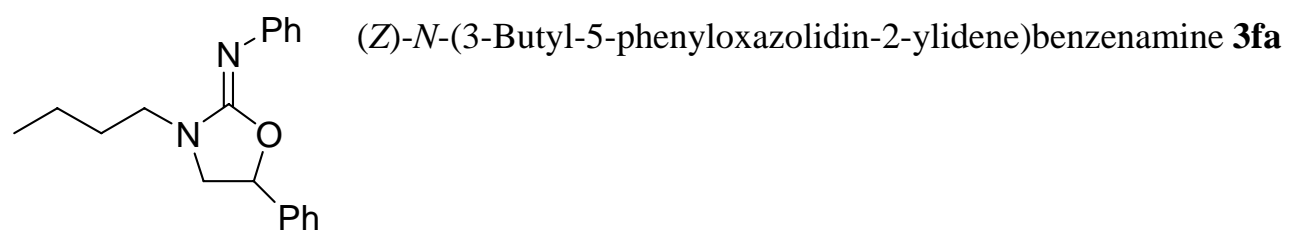

yellow oil; ${ }^{1} \mathrm{H}$ NMR (270 MHz, $\left.\mathrm{CDCl}_{3}\right)$ 7.22-7.01 (m, 9H), 6.80 (tt, $\left.J=7.8,1.6 \mathrm{~Hz}, 1 \mathrm{H}\right), 5.24$ (t, $J=$ $7.6 \mathrm{~Hz}, 1 \mathrm{H}), 3.61$ (t, $J=9.3 \mathrm{~Hz}, 1 \mathrm{H}), 3.32-3.14(\mathrm{~m}, 3 \mathrm{H}), 1.51-1.40(\mathrm{~m}, 2 \mathrm{H}), 1.27-1.13(\mathrm{~m}, 2 \mathrm{H}), 0.85$ (t, $J=8.1 \mathrm{~Hz}, 3 \mathrm{H}) ;{ }^{13} \mathrm{C}$ NMR (150 MHz, $\left.\mathrm{CDCl}_{3}\right)$ 152.7, 140.6, 147.9, 139.0, 128.7, 128.6, 128.4, 125.7, 123.6, 121.7, 77.1, 53.1, 44.8, 29.4, 20.0, 13.9; IR (neat) 3060, 3031, 2958, 2926, 2862, 1681, 1591, 1493, 1455, 1425, 1370, 1330, 1287, 1230, $1195 \mathrm{~cm}^{-1}$; Anal. Calcd for $\mathrm{C}_{19} \mathrm{H}_{22} \mathrm{~N}_{2} \mathrm{O}$ : C, 77.52; H, 7.53; N, 9.52. Found: C, 77.35; H, 7.53; N, 9.46.

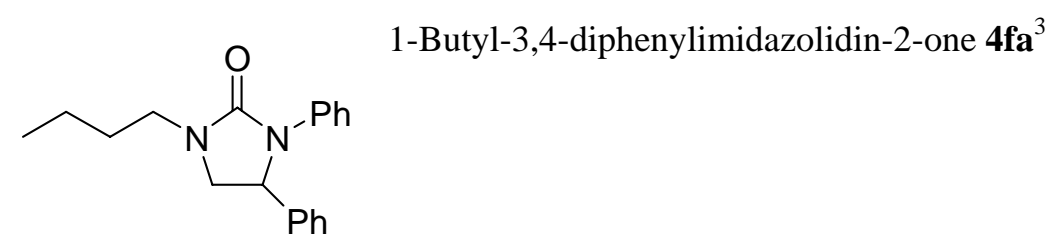

white solid; ${ }^{1} \mathrm{H}$ NMR (600 MHz, $\left.\mathrm{CDCl}_{3}\right) 7.42$ (d, $\left.J=8.4 \mathrm{~Hz}, 2 \mathrm{H}\right), 7.33-7.25$ (m, 5H), 7.19 (t, $J=9.0$ Hz, 2H), 6.94 (t, $J=7.2 \mathrm{~Hz}, 1 \mathrm{H}), 5.18$ (dd, $J=9.0,5.4 \mathrm{~Hz}, 1 \mathrm{H}), 3.87(\mathrm{t}, J=9.0 \mathrm{~Hz}, 1 \mathrm{H}), 3.32$ (t, $J=$ $7.2 \mathrm{~Hz}, 2 \mathrm{H}$ ), 3.21 (dd, $J=8.4,6.0 \mathrm{~Hz}, 1 \mathrm{H}), 1.54-1.49$ (m, 2H), 1.39-1.33 (m, 2H), 0.93 (t, $J=7.2 \mathrm{~Hz}$, $3 \mathrm{H})$; ${ }^{13} \mathrm{C}$ NMR (150 MHz, $\mathrm{CDCl}_{3}$ ) 158.3, 140.7, 139.4, 129.1, 128.5, 128.0, 126.0, 122.7, 119.6, 57.6, 51.5, 43.6, 29.5, 19.9, 13.7 . 


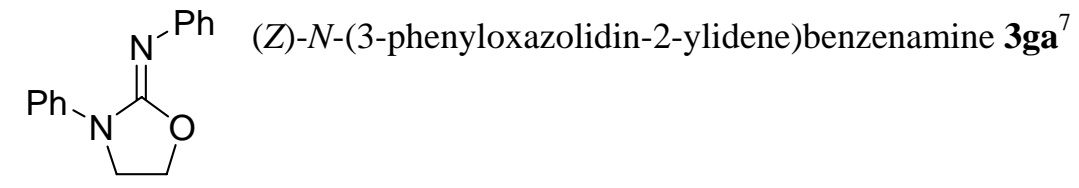

white solid, mp 116-117 ${ }^{\circ} \mathrm{C}_{\text {(lit. }}{ }^{7} 115-116{ }^{\circ} \mathrm{C}$ ); ${ }^{1} \mathrm{H}$ NMR $\left(600 \mathrm{MHz}, \mathrm{CDCl}_{3}\right) 7.78$ (d, $J=8.4 \mathrm{~Hz}$, 2H), 7.38 (t, $J=8.4 \mathrm{~Hz}, 2 \mathrm{H}), 7.28$ (t, $J=7.2 \mathrm{~Hz}, 2 \mathrm{H}$ ), 7.11-7.09 (m, 3H), 7.01 (t, $J=7.2 \mathrm{~Hz}, 1 \mathrm{H}$ ), 4.45 (t, $J=7.8 \mathrm{~Hz}, 2 \mathrm{H}), 4.05$ (t, $J=7.8 \mathrm{~Hz}, 2 \mathrm{H}) ;{ }^{13} \mathrm{C} \mathrm{NMR}\left(150 \mathrm{MHz}, \mathrm{CDCl}_{3}\right)$ 149.1, 147.4, 139.7, $128.8,128.5,123.2,123.0,122.5,118.8,63.7,46.4$.

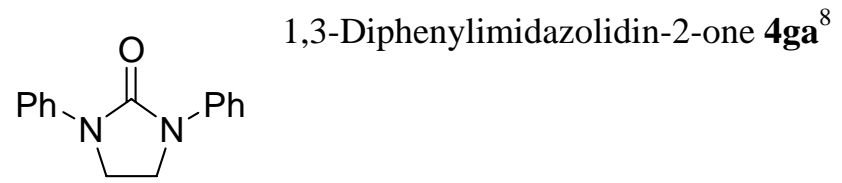

white solid; ${ }^{1} \mathrm{H}$ NMR (600 MHz, $\left.\mathrm{CDCl}_{3}\right) 7.60$ (d, $J=7.8 \mathrm{~Hz}, 4 \mathrm{H}$ ), 7.38 (t, $J=7.8 \mathrm{~Hz}, 4 \mathrm{H}$ ), 7.10 (t, $J$ = $7.2 \mathrm{~Hz}, 2 \mathrm{H}), 3.99$ (s, 4H); ${ }^{13} \mathrm{C} \mathrm{NMR}\left(150 \mathrm{MHz}, \mathrm{CDCl}_{3}\right)$ 140.0, 128.9, 123.1, 118.1, 42.0 .<smiles>c1ccc(CN=C2OCCN2Cc2ccccc2)cc1</smiles>

(Z)- $N$-(3-benzyloxazolidin-2-ylidene)(phenyl)methanamine 3ab

yellow oil; ${ }^{1} \mathrm{H}$ NMR (300 MHz, $\mathrm{CDCl}_{3}$ ) 7.34-7.12 (m, 10H), 4.45 (s, 2H), 4.41 (s, 2H), 4.09 (t, $J=$ $7.2 \mathrm{~Hz}, 2 \mathrm{H}), 3.12$ (t, $J=7.8 \mathrm{~Hz}, 2 \mathrm{H}) ;{ }^{13} \mathrm{C}$ NMR $\left(75 \mathrm{MHz}, \mathrm{CDCl}_{3}\right)$ 154.9, 142.2, 136.8, 128.4, 128.1, 127.9, 127.3, 127.2, 125.9, 64.1, 49.8, 49.3, 45.7; IR (neat) 3060, 3026, 2905, 2852, 1697, 1603, 1583, 1539, 1493, 1452, 1351, 1259, $1192 \mathrm{~cm}^{-1}$. HRMS Calcd for $\mathrm{C}_{17} \mathrm{H}_{18} \mathrm{~N}_{2} \mathrm{O}$ : 266.1419. Found: 266.1415.<smiles>COc1ccc(/N=C2\OCCN2Cc2ccccc2)cc1</smiles>

(Z)- $N$-(3-benzyloxazolidin-2-ylidene)-4-methoxybenzenamine 3ac

light yellow solid, mp 84-85 ${ }^{\circ} \mathrm{C}$; ${ }^{1} \mathrm{H}$ NMR (300 MHz, CDCl $)$ ) 7.38-7.26 (m, 6H), 7.02 (d, $J=8.1 \mathrm{~Hz}$, 2H), 6.80 (d, $J=8.7 \mathrm{~Hz}, 2 \mathrm{H}$ ), 4.56 (s, 2H), 4.28 (t, $J=8.1 \mathrm{~Hz}, 2 \mathrm{H}$ ), 3.76 (s, 3H), 3.34 (t, $J=7.5 \mathrm{~Hz}$, 2H); ${ }^{13} \mathrm{C}$ NMR (150 MHz, $\mathrm{CDCl}_{3}$ ) 154.8, 153.1, 140.9, 136.8, 128.6, 128.3, 127.6, 124.1, 113.9, 64.5, 55.4, 49.3, 45.3; IR (KBr) 3059, 3000, 2946, 2830, 1680, 1573, 1507, 1485, 1454, 1435, 1356, 1293, 1266, 1241, 1203, 1180, 1124, $1105 \mathrm{~cm}^{-1}$; Anal. Calcd for $\mathrm{C}_{17} \mathrm{H}_{18} \mathrm{~N}_{2} \mathrm{O}_{2}$ : C, 72.32; H, 6.43; N, 9.92. Found: C, 72.20; H, 6.65; N, 9.82. 
<smiles>CC(=O)c1ccc(/N=C2\OCCN2Cc2ccccc2)cc1</smiles>

white solid, mp 80-81 ${ }^{\circ} \mathrm{C}$; ${ }^{1} \mathrm{H}$ NMR (600 MHz, $\left.\mathrm{CDCl}_{3}\right) 7.87$ (d, $\left.J=8.4 \mathrm{~Hz}, 2 \mathrm{H}\right), 7.36-7.29$ (m, 5H), 7.14 (d, $J=8.4 \mathrm{~Hz}, 2 \mathrm{H}$ ), 4.59 (s, 2H), 4.34 (t, $J=7.2 \mathrm{~Hz}, 2 \mathrm{H}$ ), 3.41 (t, $J=7.8 \mathrm{~Hz}, 2 \mathrm{H}$ ), 2.54 (s, 3H); ${ }^{13} \mathrm{C}$ NMR (150 MHz, $\mathrm{CDCl}_{3}$ ) 153.8, 153.1, 136.3, 131.0, 129.4, 128.7, 128.3, 127.8, 123.4, 64.8, 49.1, 45.1, 26.4; IR (KBr) 3063, 3029, 2911, 1671, 1590, 1556, 1486, 1451, 1427, 1411, 1380, 1354 , 1294, 1271, 1205, 1172, 1116, 1089, 1065, $1025 \mathrm{~cm}^{-1}$; Anal. Calcd for $\mathrm{C}_{18} \mathrm{H}_{18} \mathrm{~N}_{2} \mathrm{O}_{2}$ : C, 73.45; $\mathrm{H}$, 6.16; N, 9.52. Found: C, 73.1; H, 6.36; N, 9.41.<smiles>c1ccc(CN2CCOC2=Nc2cccc3ccccc23)cc1</smiles>

(Z)- $N$-(3-benzyloxazolidin-2-ylidene)naphthalen-1-amine 3ae

yellow solid, mp 85-86 ${ }^{\circ} \mathrm{C} ;{ }^{1} \mathrm{H}$ NMR $\left(270 \mathrm{MHz}, \mathrm{CDCl}_{3}\right) 8.24$ (t, $\left.J=5.1 \mathrm{~Hz}, 1 \mathrm{H}\right), 7.78$ (t, $J=4.3 \mathrm{~Hz}$, 1H), 7.51-7,24 (m, 9H), 7.19 (d, $J=6.2 \mathrm{~Hz}, 1 \mathrm{H}), 4.74$ (s, 2H), 4.29 (t, $J=7.0 \mathrm{~Hz}, 2 \mathrm{H}$ ), 3.43 (t, $J=$ $7.8 \mathrm{~Hz}, 2 \mathrm{H}) ;{ }^{13} \mathrm{C} \mathrm{NMR}\left(150 \mathrm{MHz}, \mathrm{CDCl}_{3}\right)$ 153.1, 144.2, 136.8, 134.3, 129.5, 128.7, 128.3, 127.7, 127.7, 125.9, 125.5, 124.7, 124.2, 122.0, 117.6, 64.5, 49.4, 45.3; IR (KBr) 3041, 2975, 2923, 2879, 2358, 1662, 1568, 1488, 1358, 1267, 1098, 1014, $938 \mathrm{~cm}^{-1}$; Anal. Calcd for $\mathrm{C}_{20} \mathrm{H}_{16} \mathrm{~N}_{2} \mathrm{O}$ : C, 79.44; $\mathrm{H}$, 6.00; N, 9.26. Found: C, 79.43; H, 5.98; N, 9.20.

\section{References}

1) Appel, R.; Kleinstück, R. Chem. Ber. 1974, 107, 5-12.

2) Elderfield, R. C.; Hageman, H. A. J. Org. Chem. 1949, 14, 605-637.

3) Baeg, J. O.; Bensimon, C.; Alper, H. J. Am. Chem. Soc. 1995, 117, 4700-4701.

4) Watson, I. D. G.; Yudin, A. K. J. Org. Chem. 2003, 68, 5160-5167.

5) Martin, A. E.; Ford, T. M.; Bulkowski, J. E. J. Org. Chem. 1982, 47, 412-415.

6) Vedejs, E.; Kendall, J. T. J. Am. Chem. Soc. 1997, 119, 6941-6942.

7) Beachell, H. C.; Ngoc Son, C. P.; Tinh, N. H. J. Org. Chem. 1972, 37, 422-425.

8) Bates, H. A.; Condulis, N.; Stein, N. L. J. Org. Chem. 1986, 51, 2228-2229. 
${ }^{1} \mathrm{H}$ and ${ }^{13} \mathrm{C}$ spectra

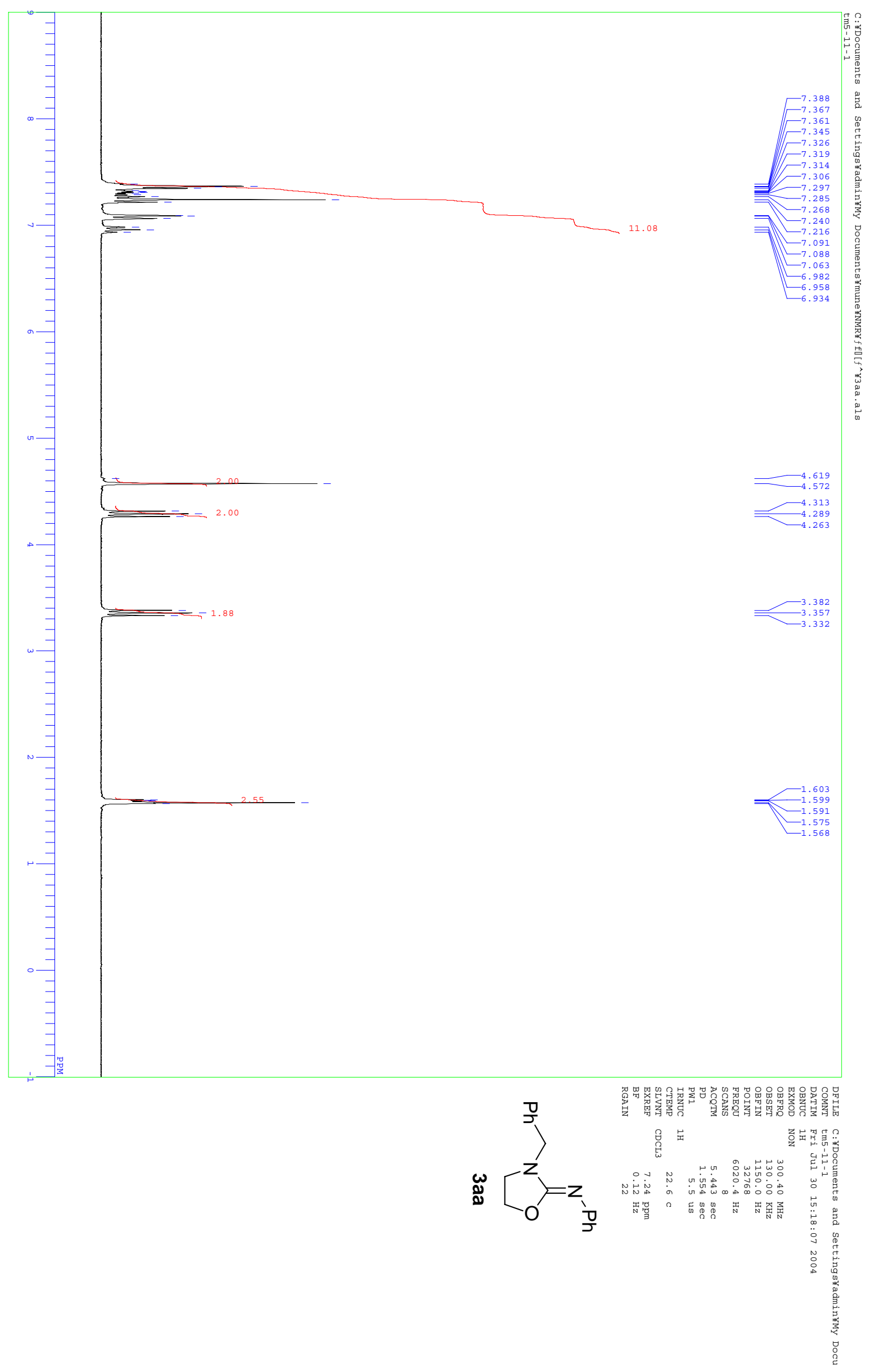




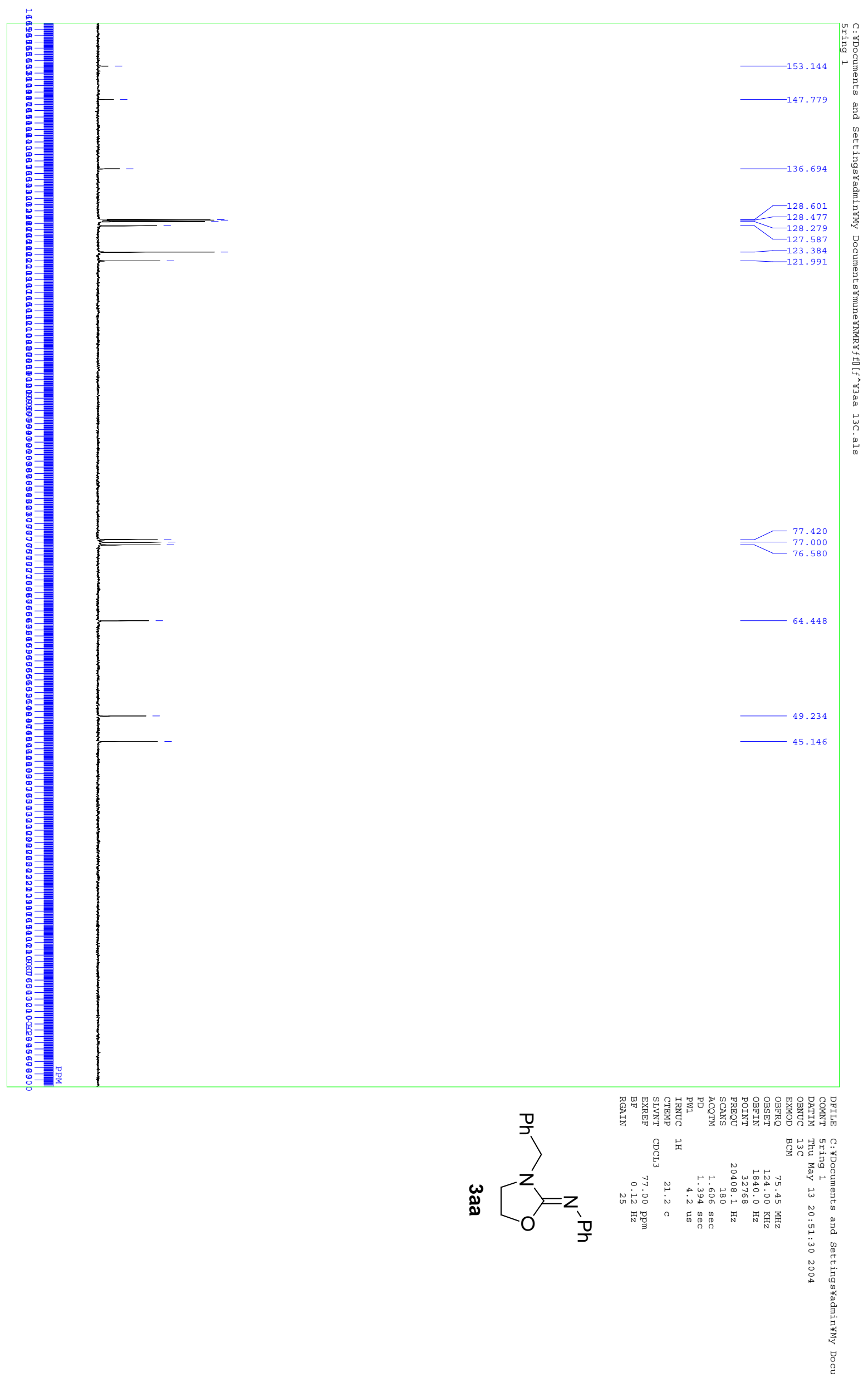




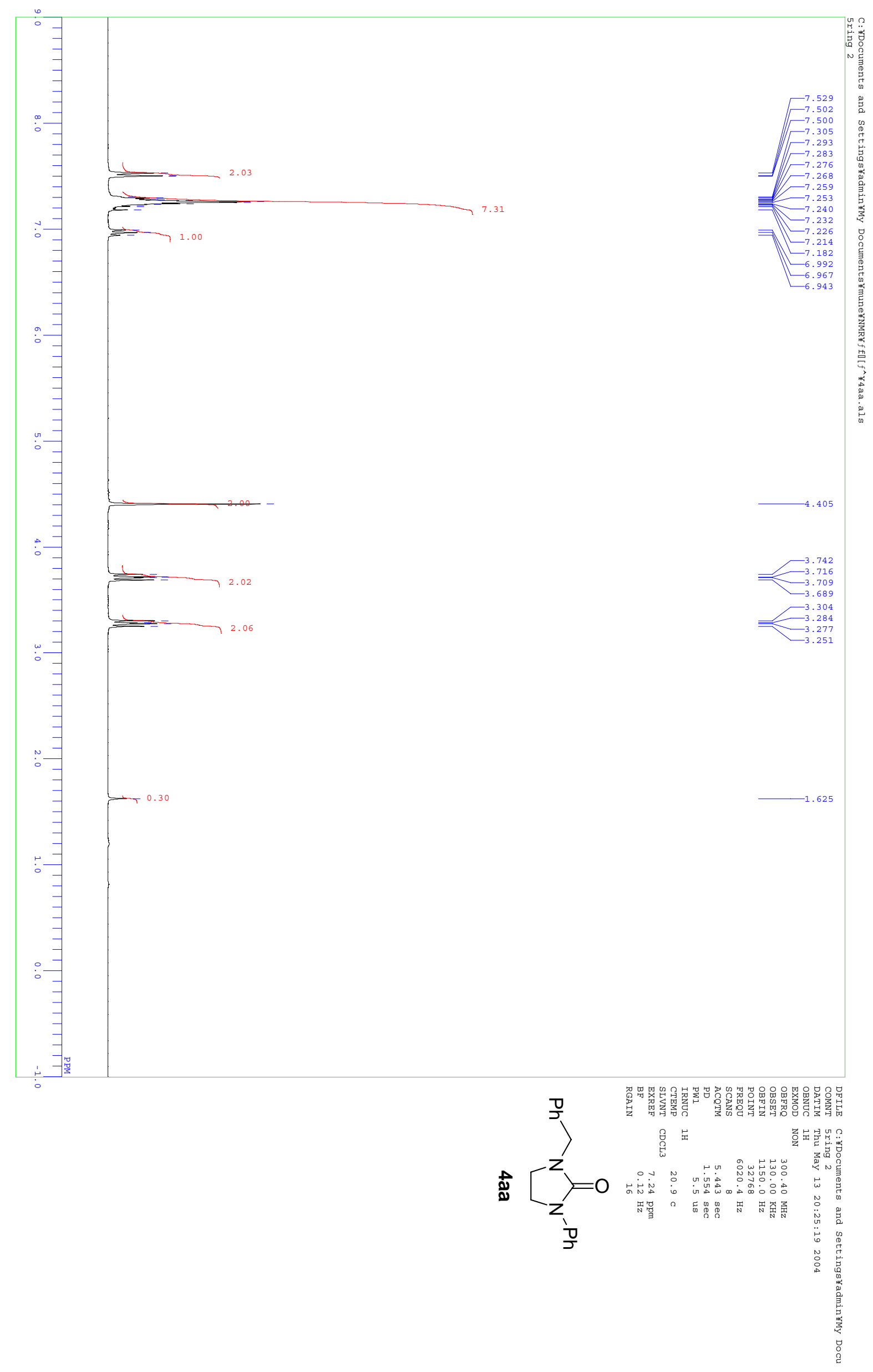



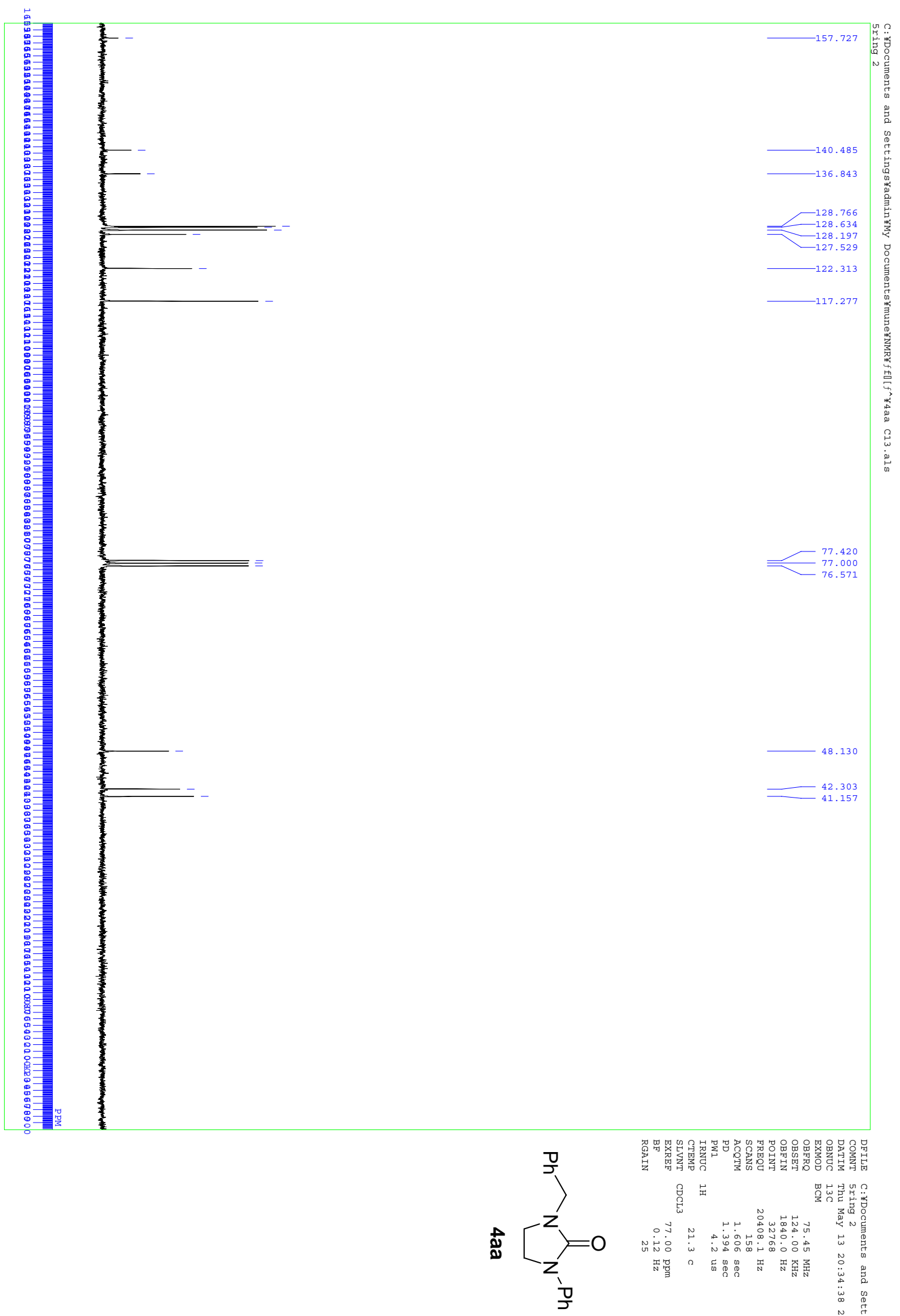

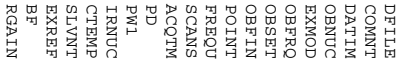

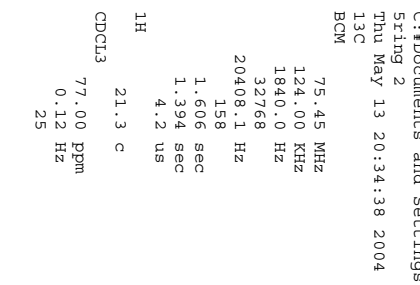




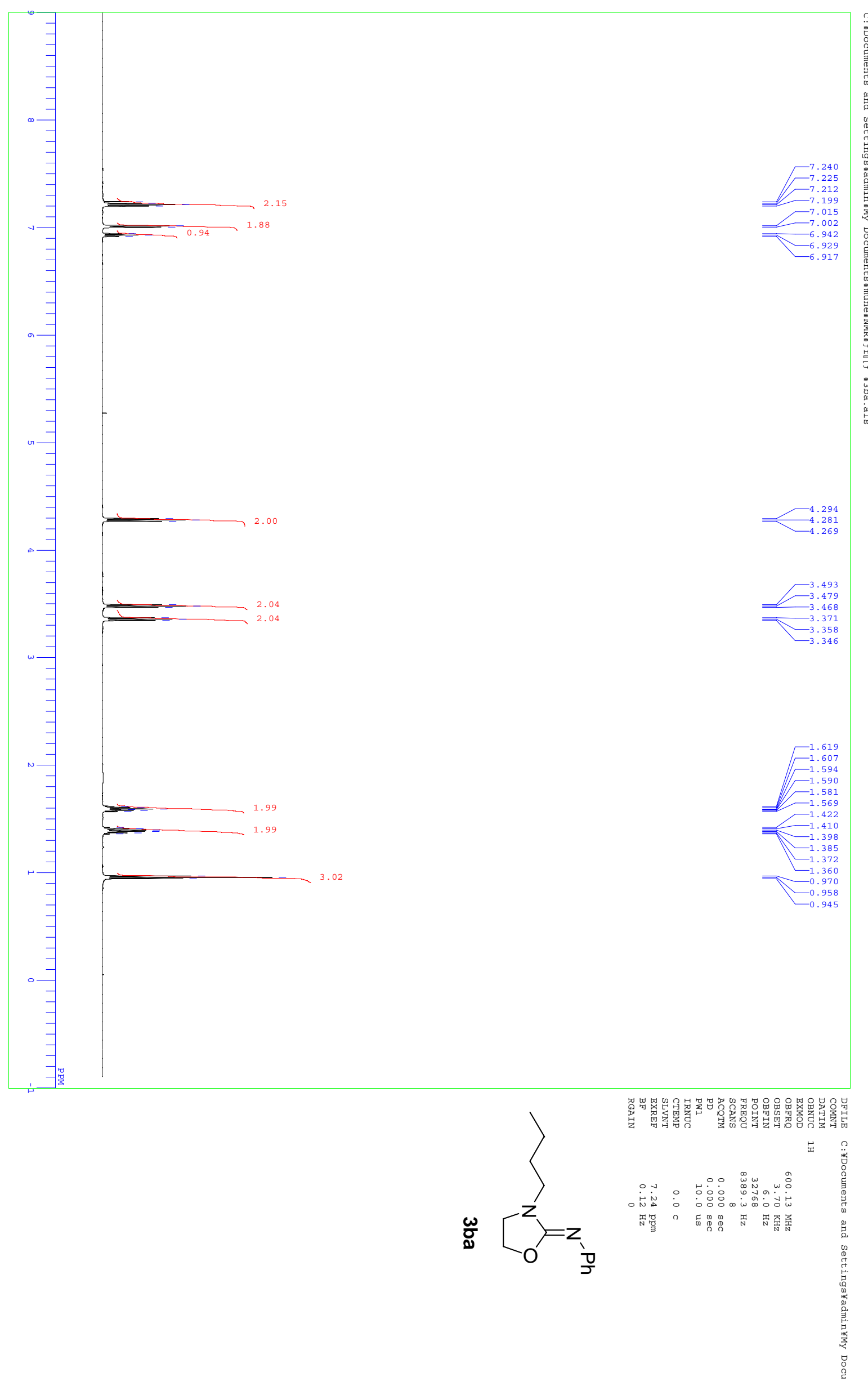




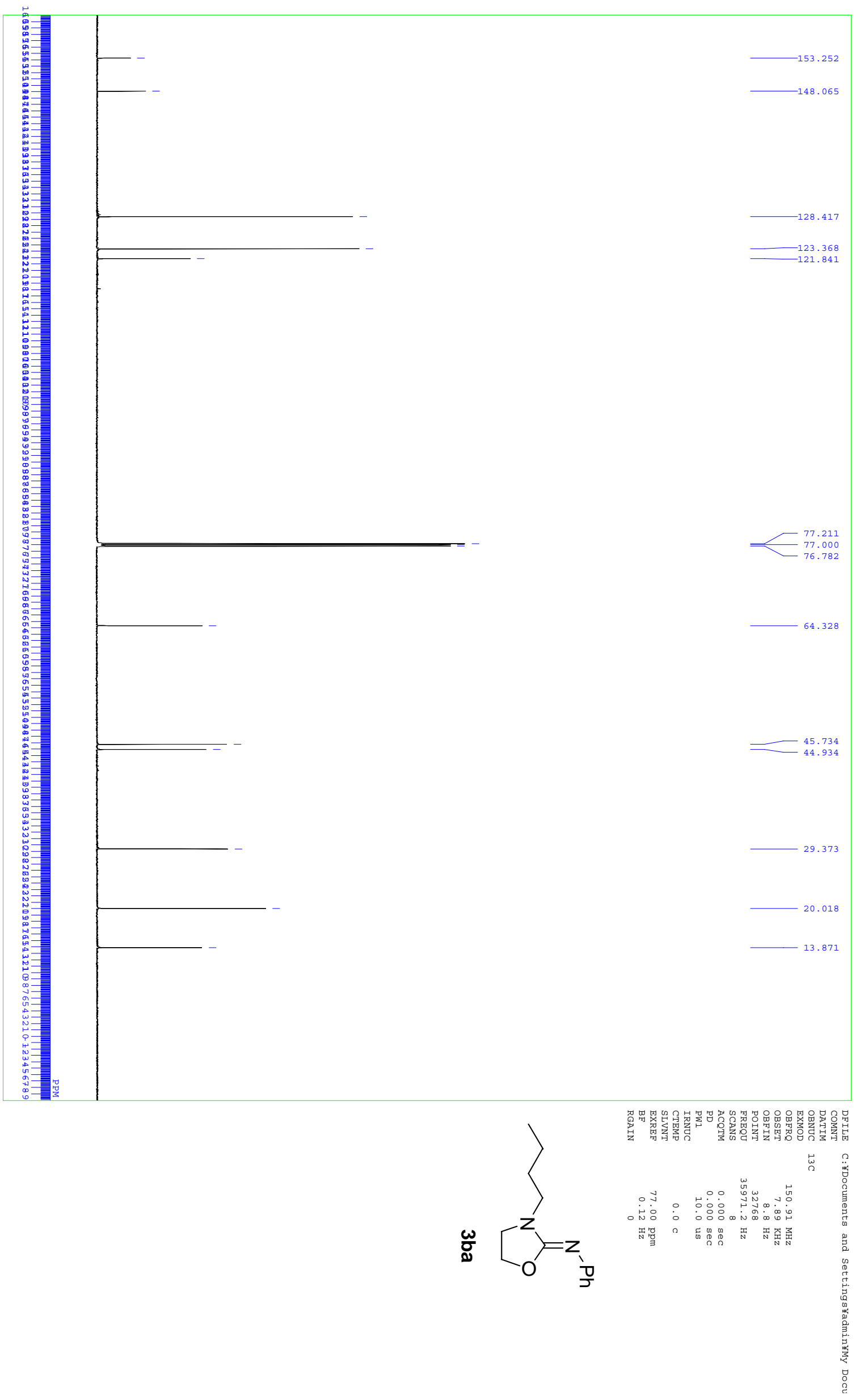

S14 


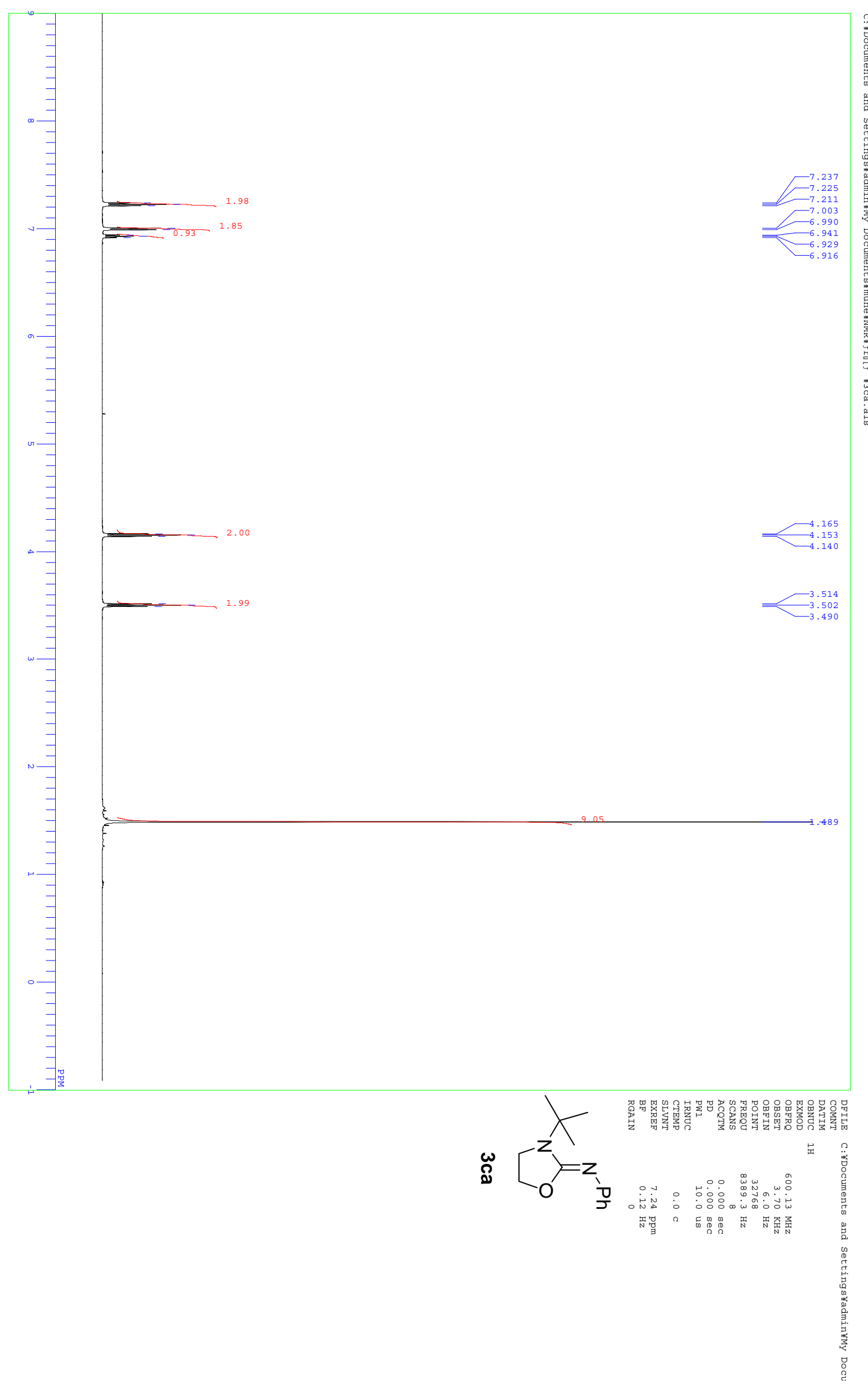




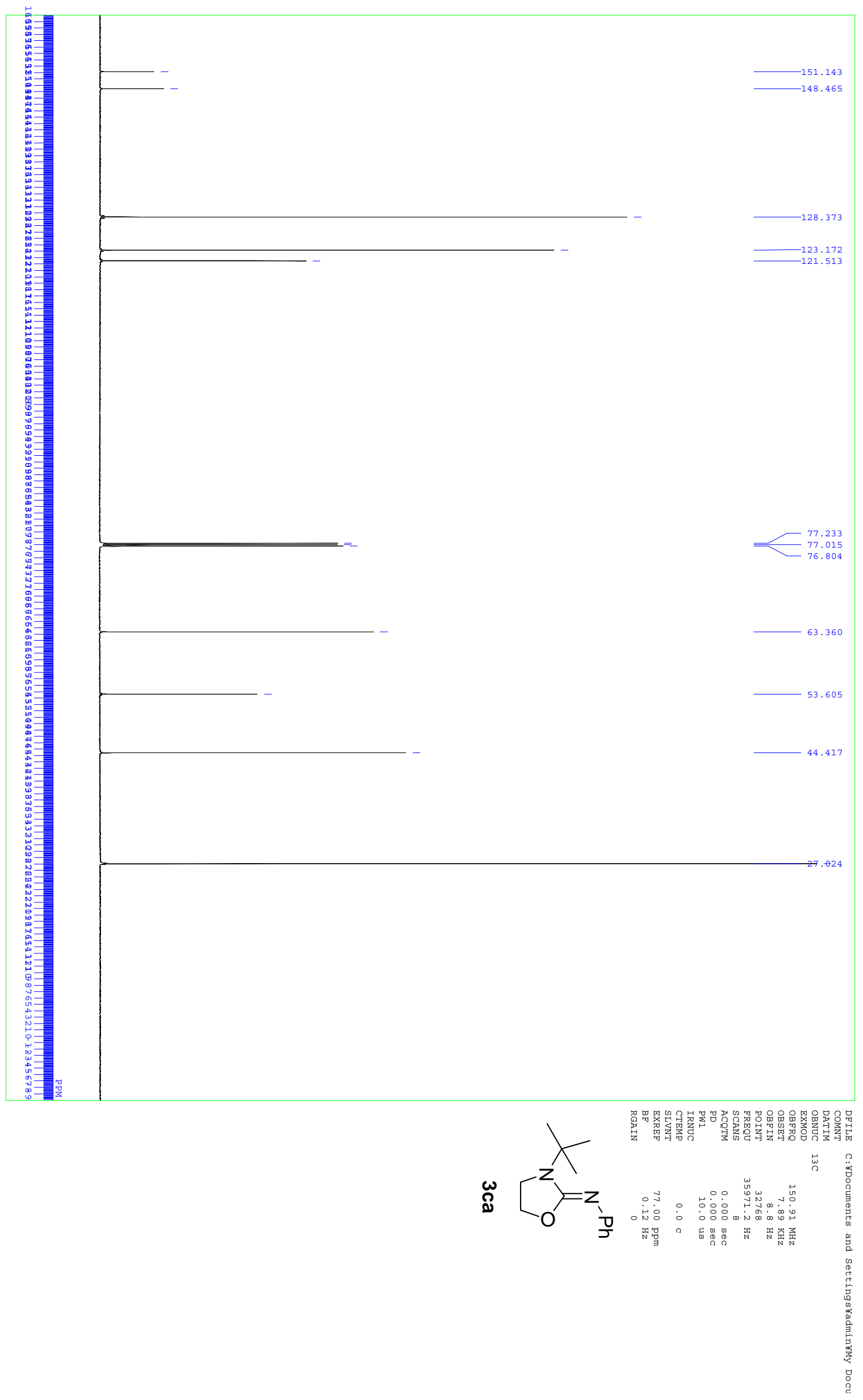

S16 


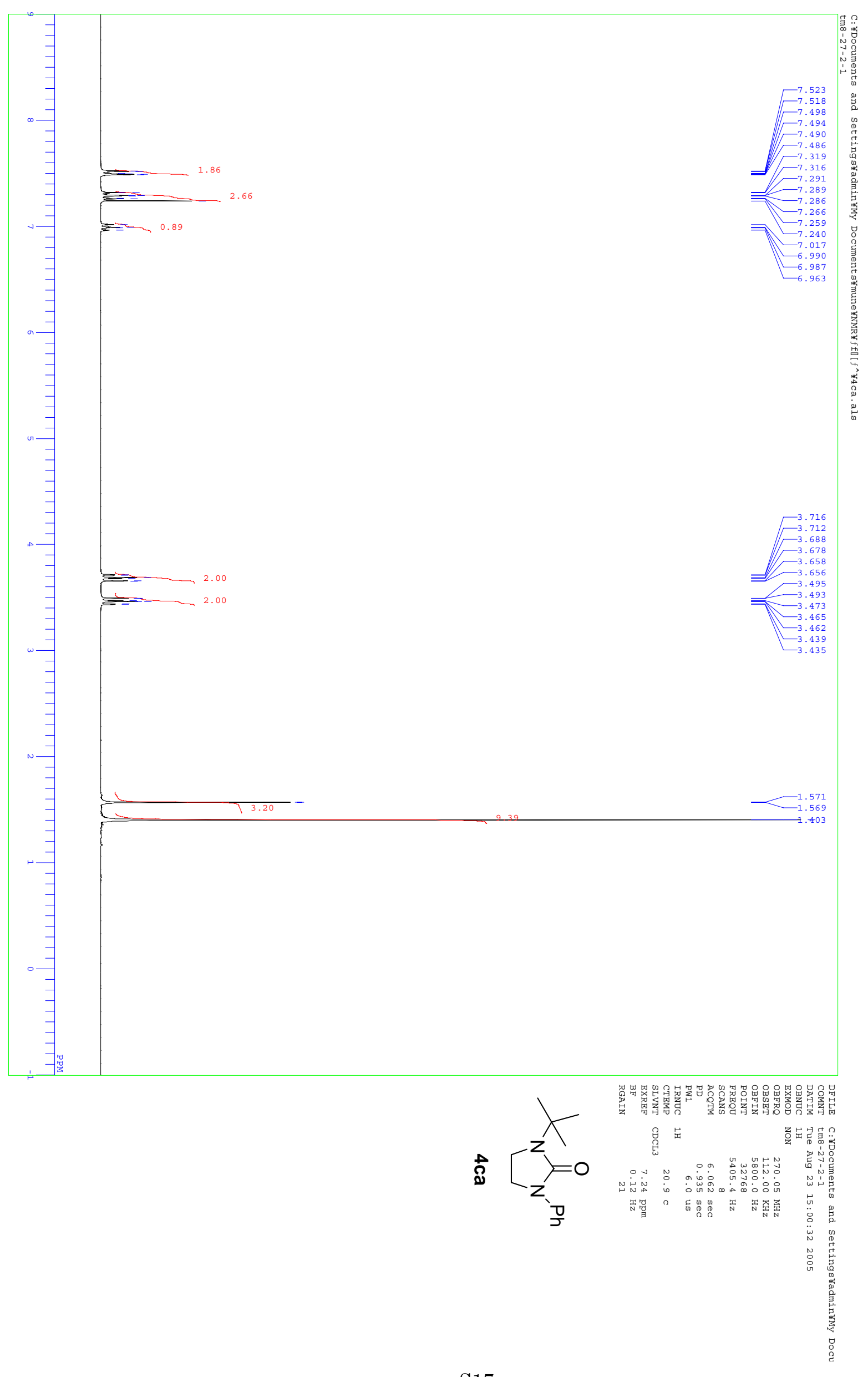



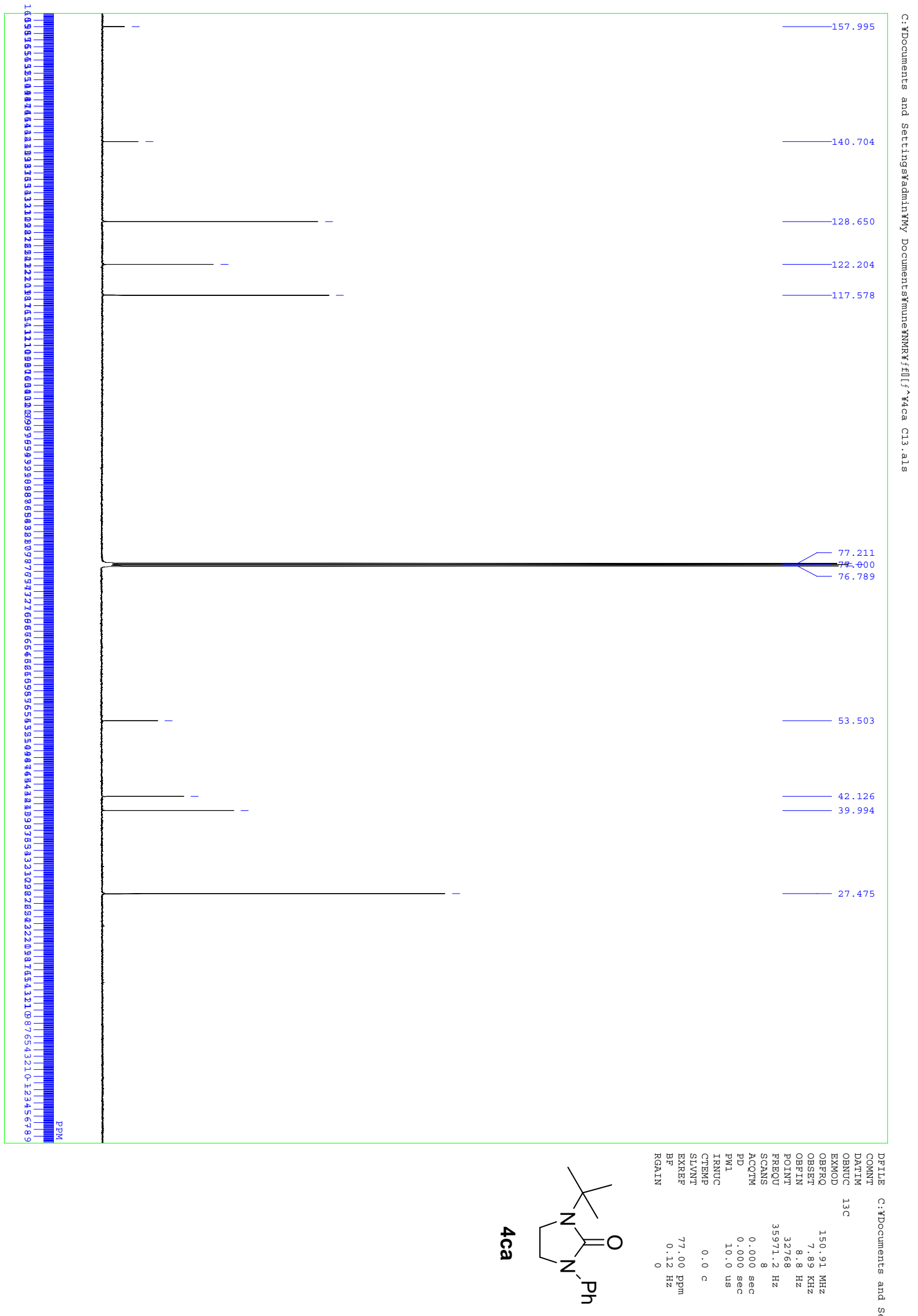


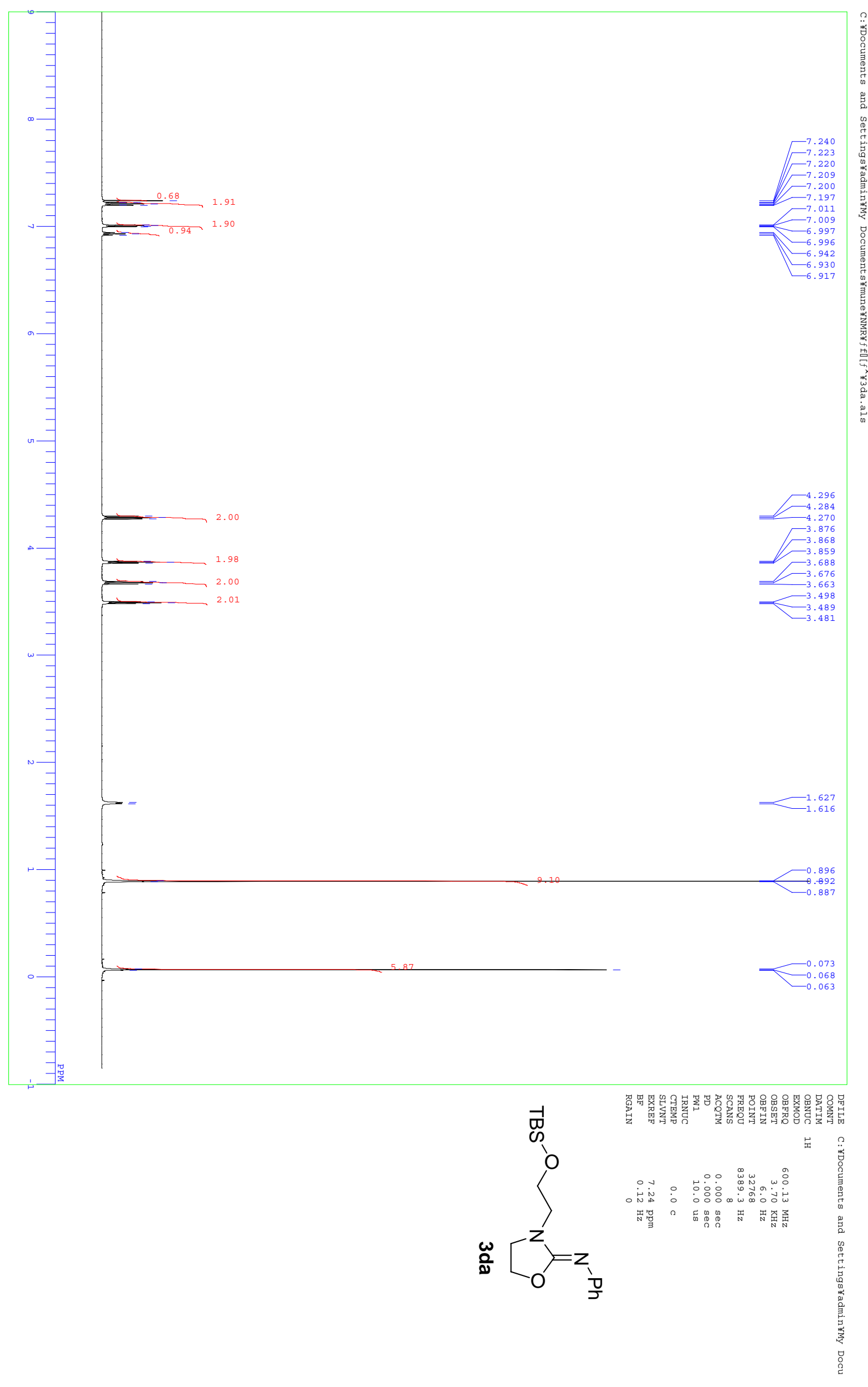




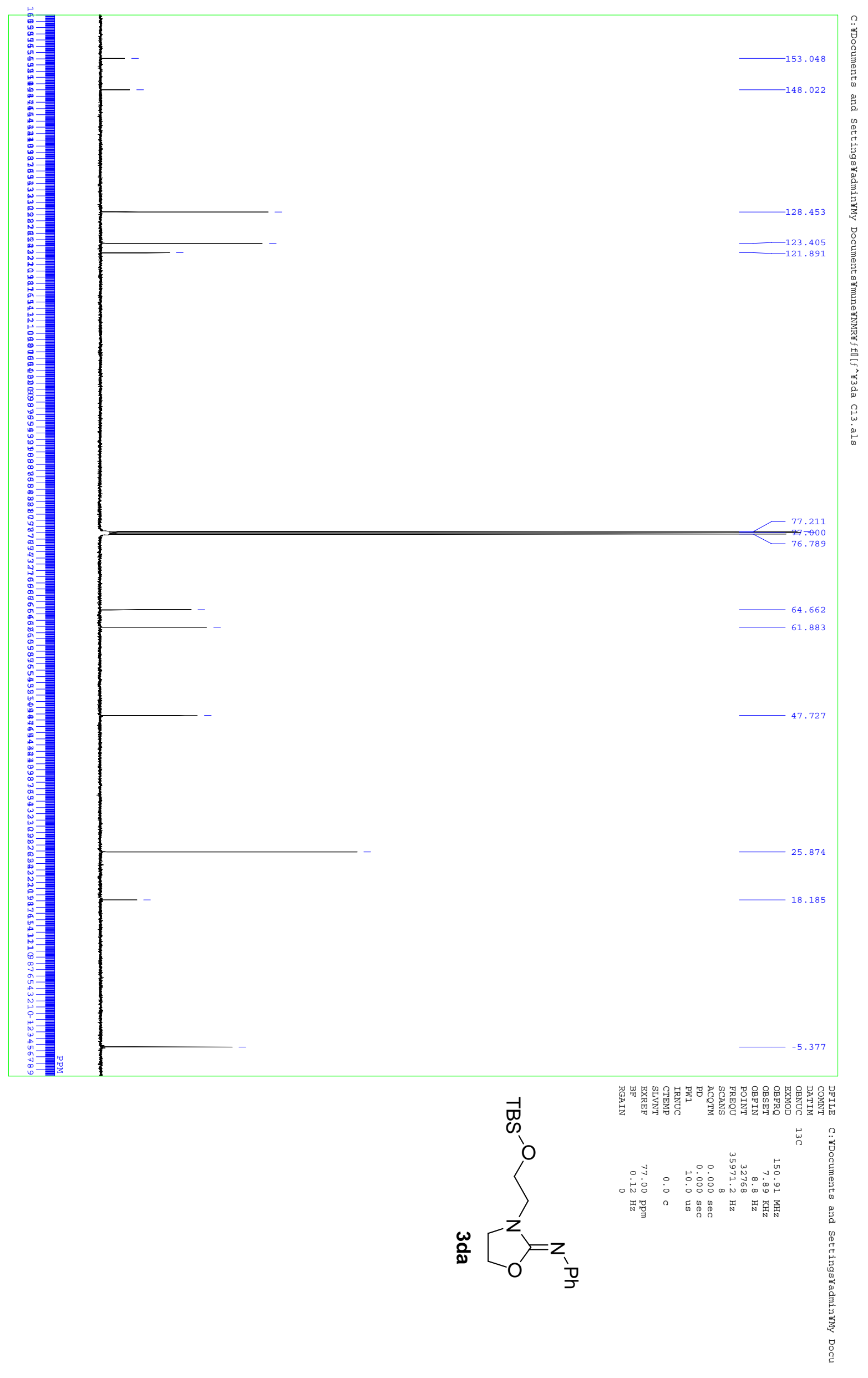




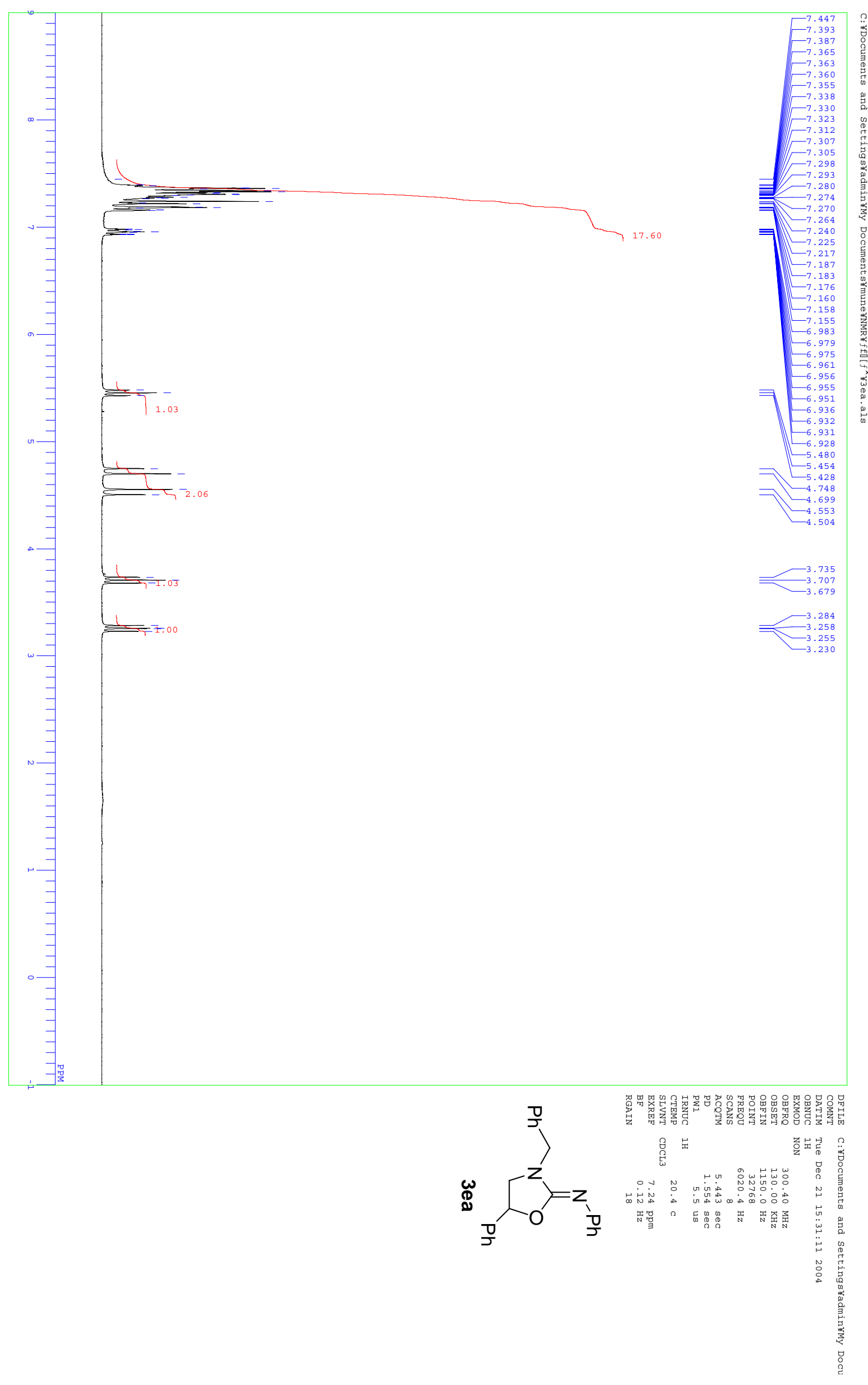




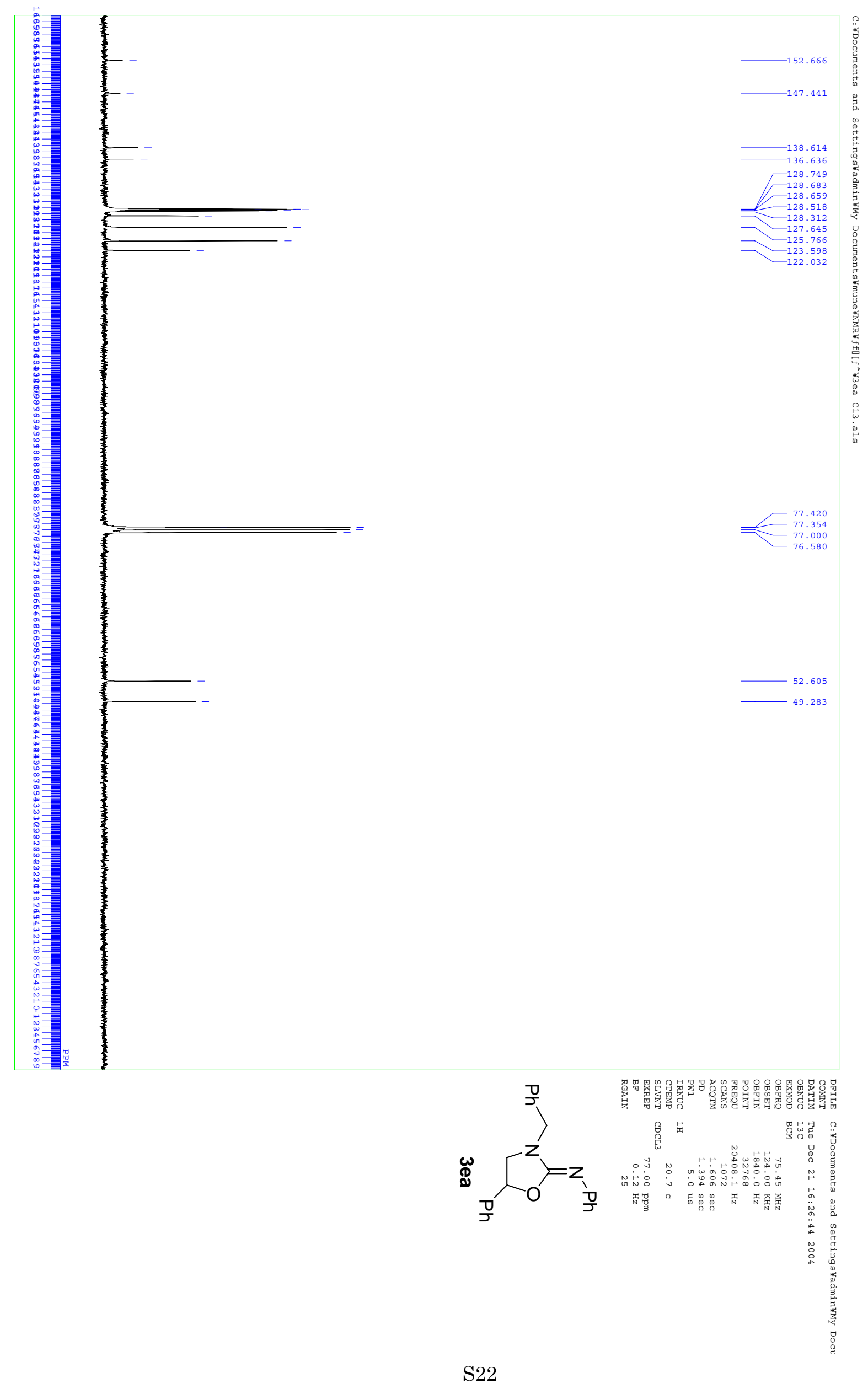




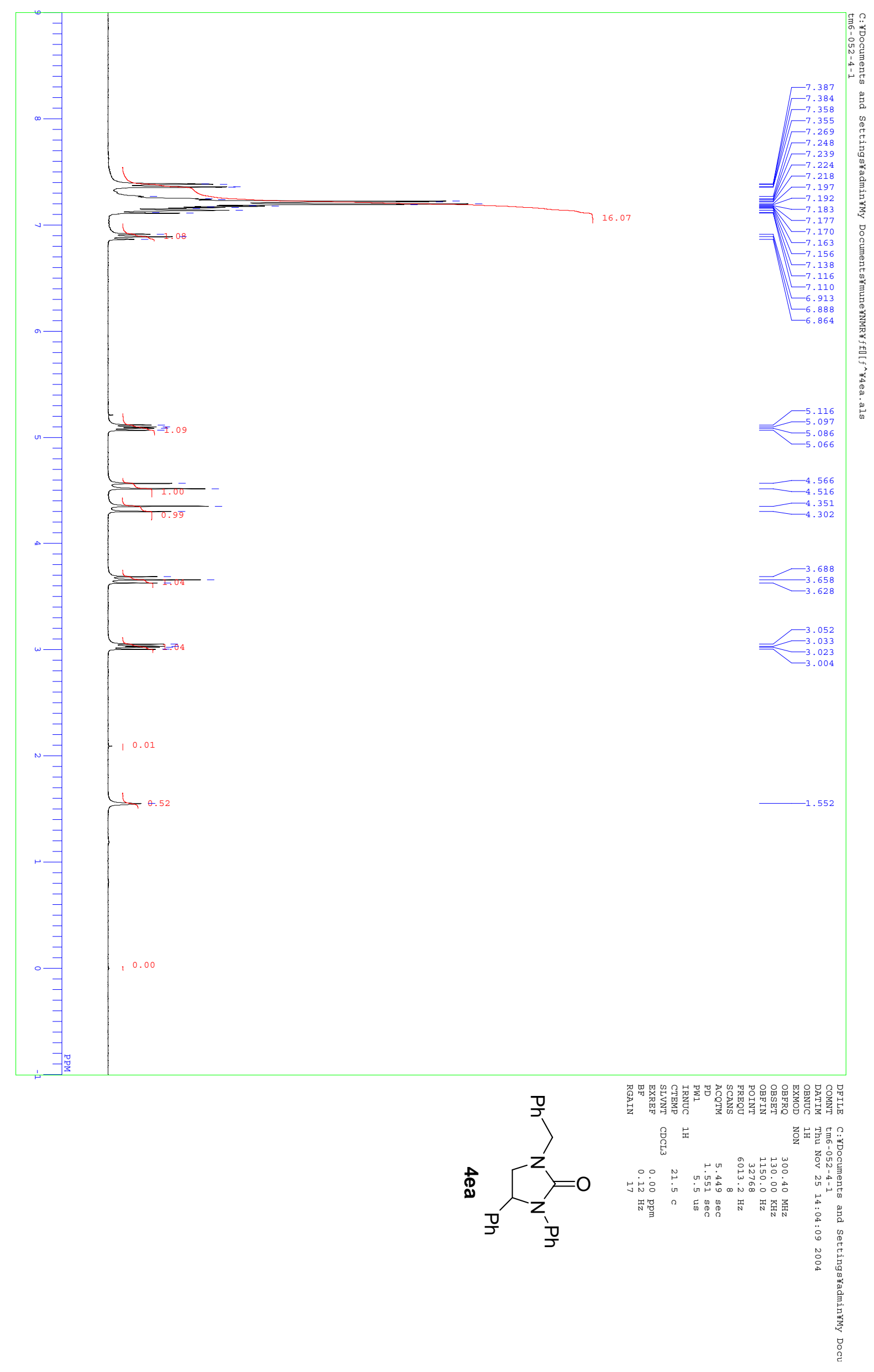




$$
1
$$




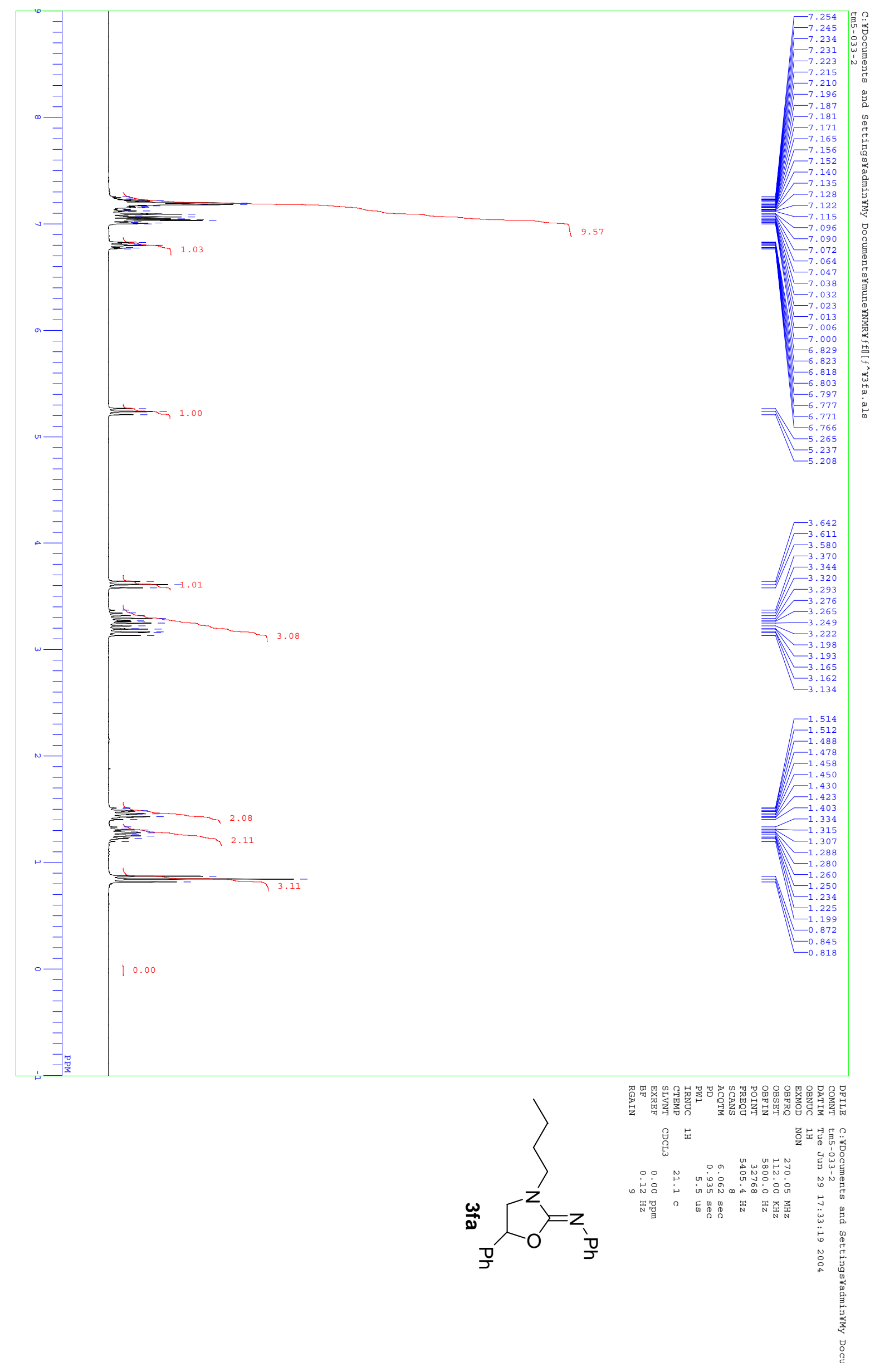




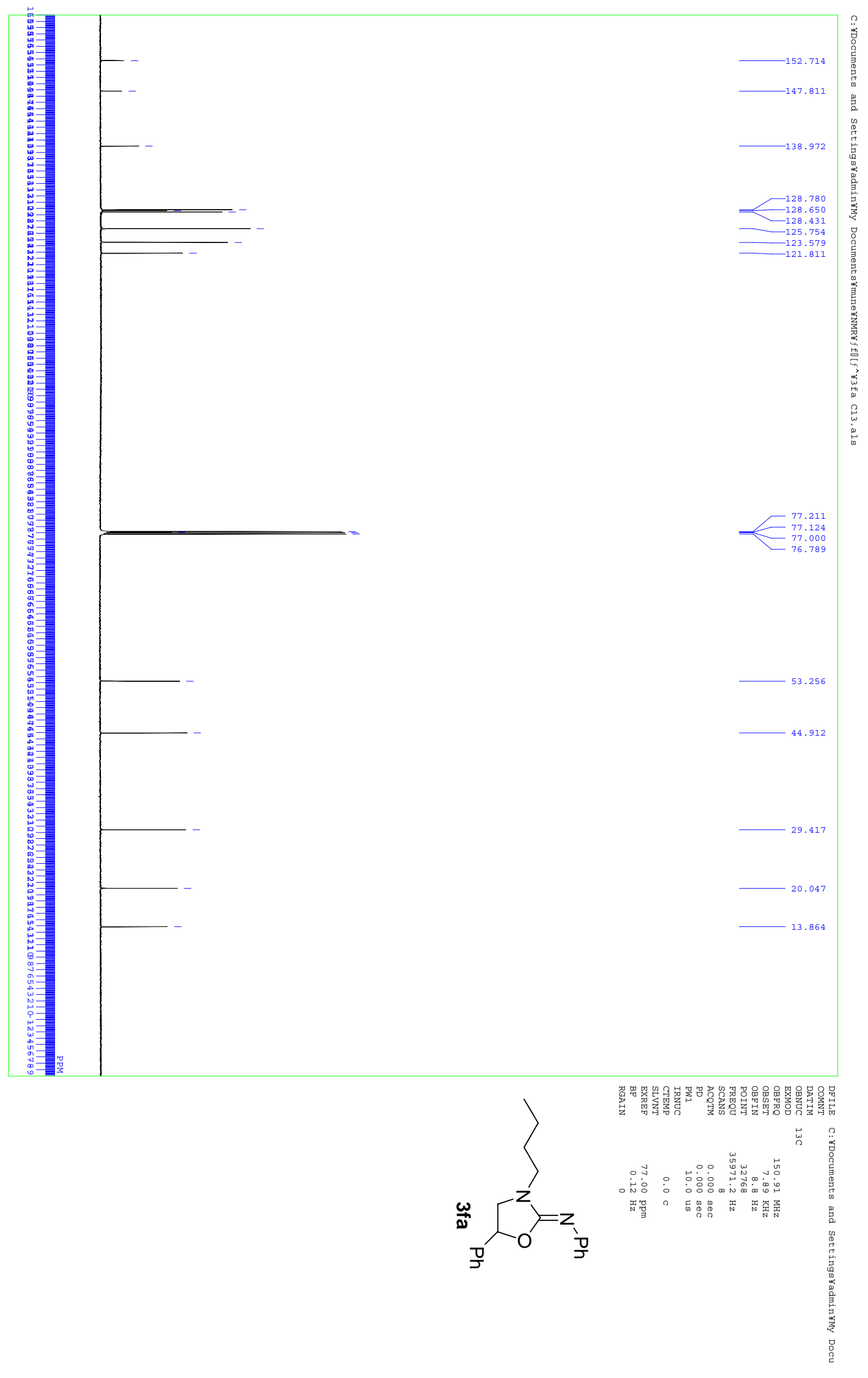




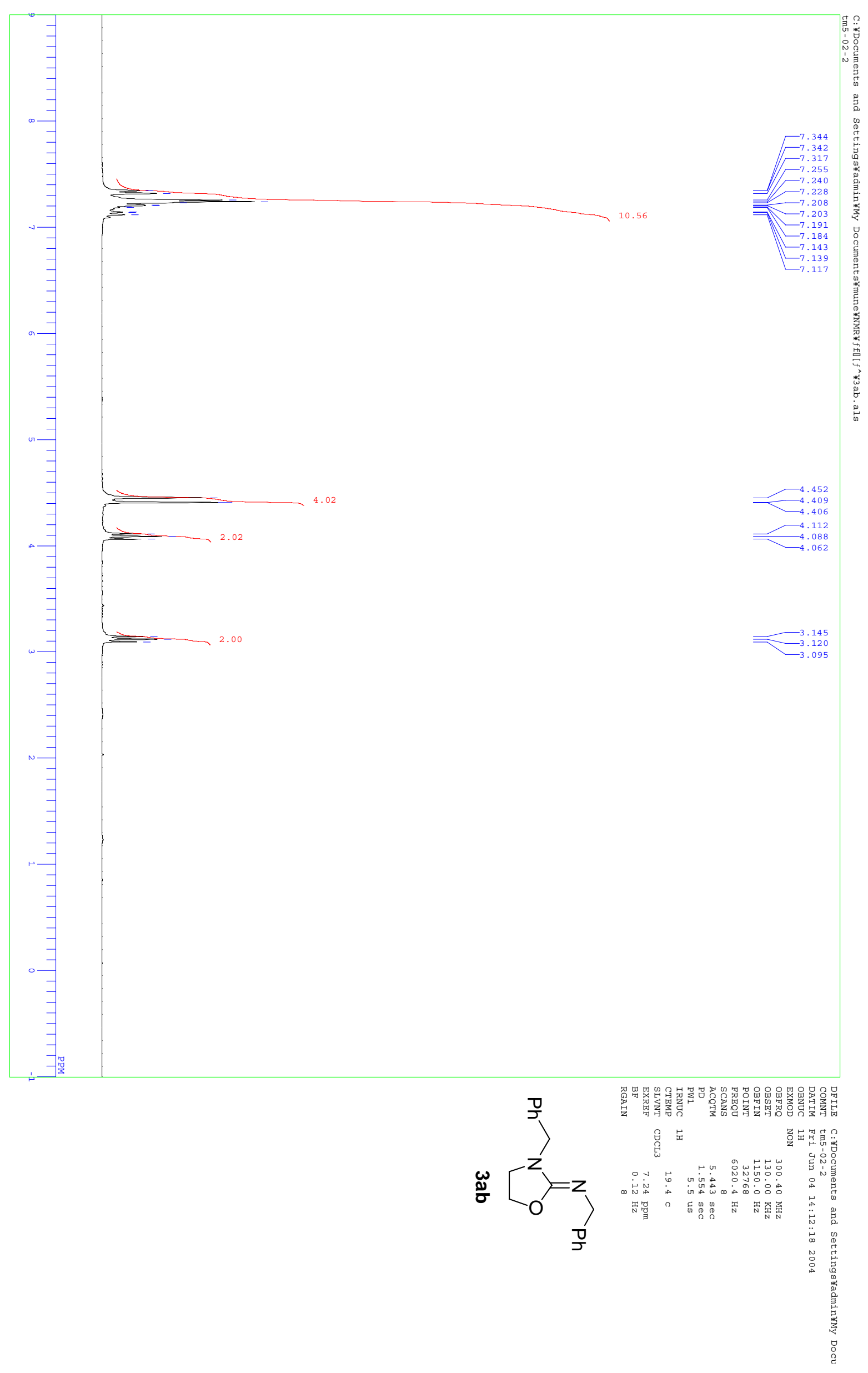



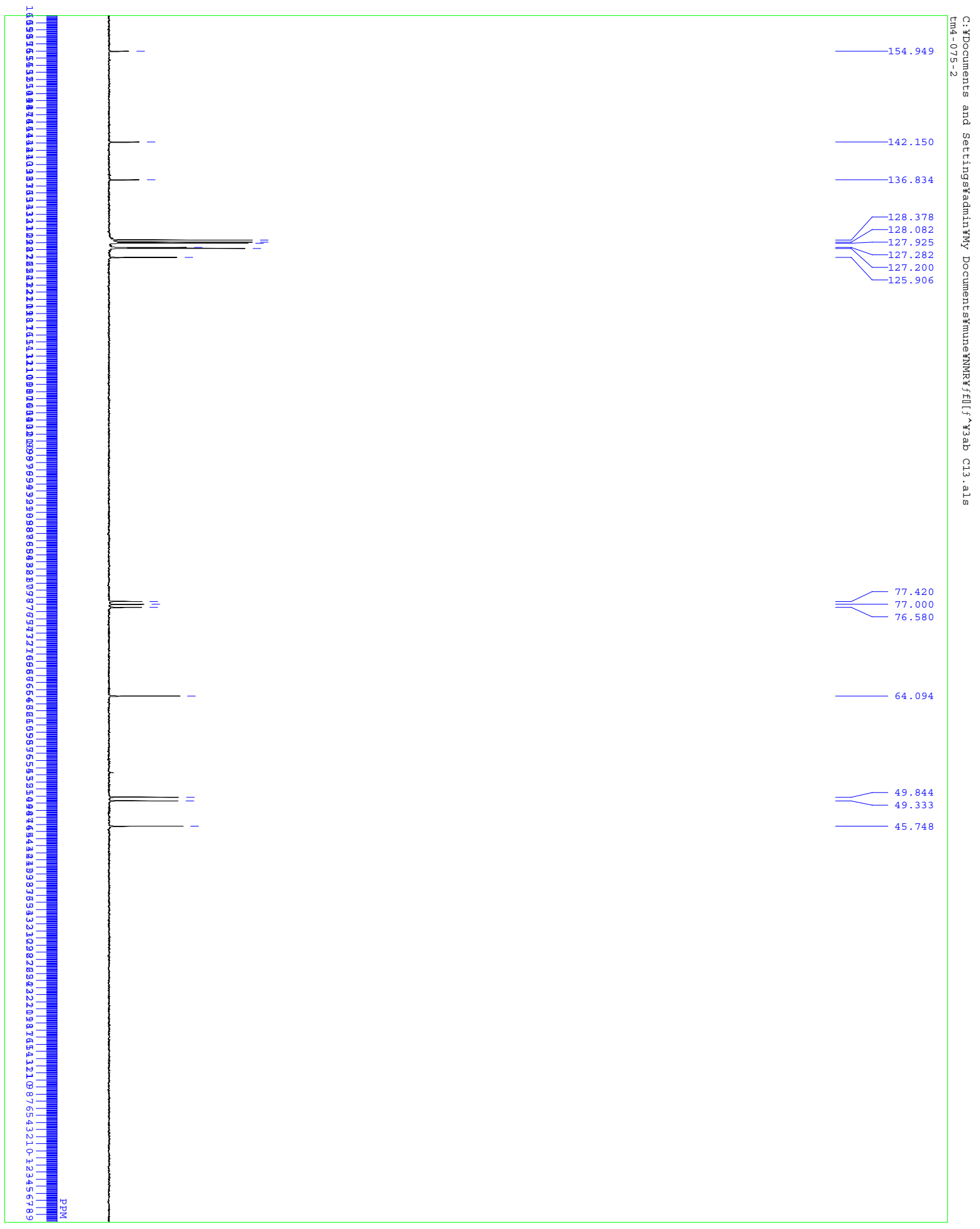

$\begin{array}{r}49.844 \\ \hline \quad{ }_{49.333} \\ \hline\end{array}$
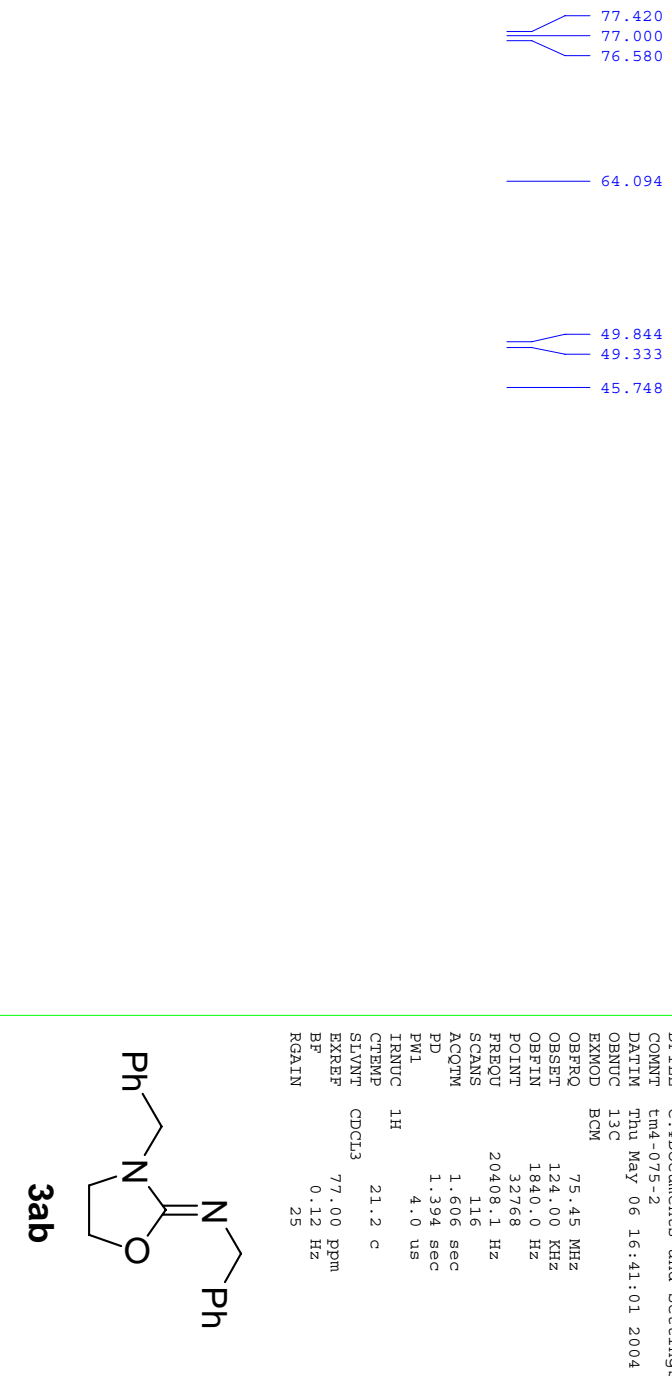


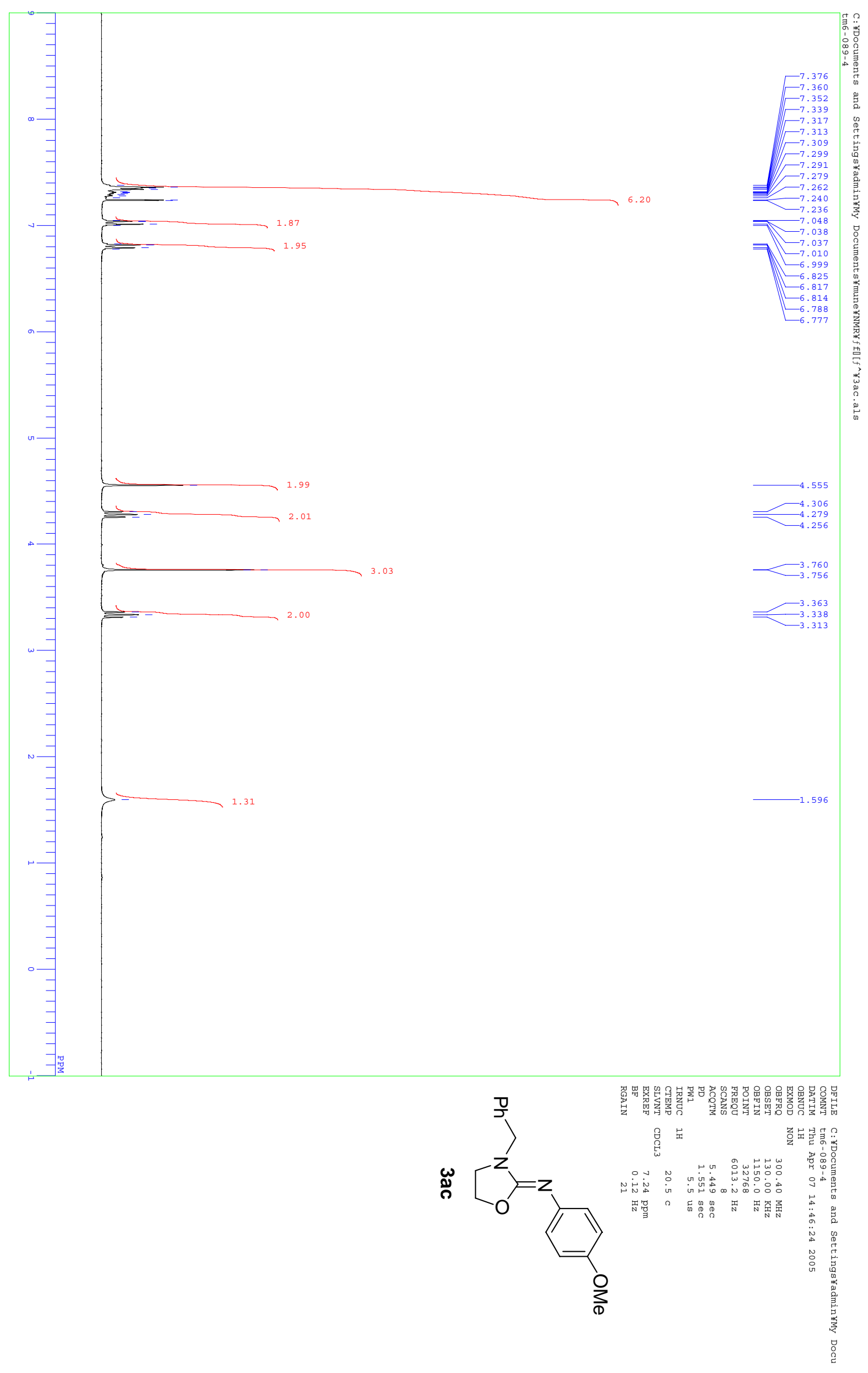




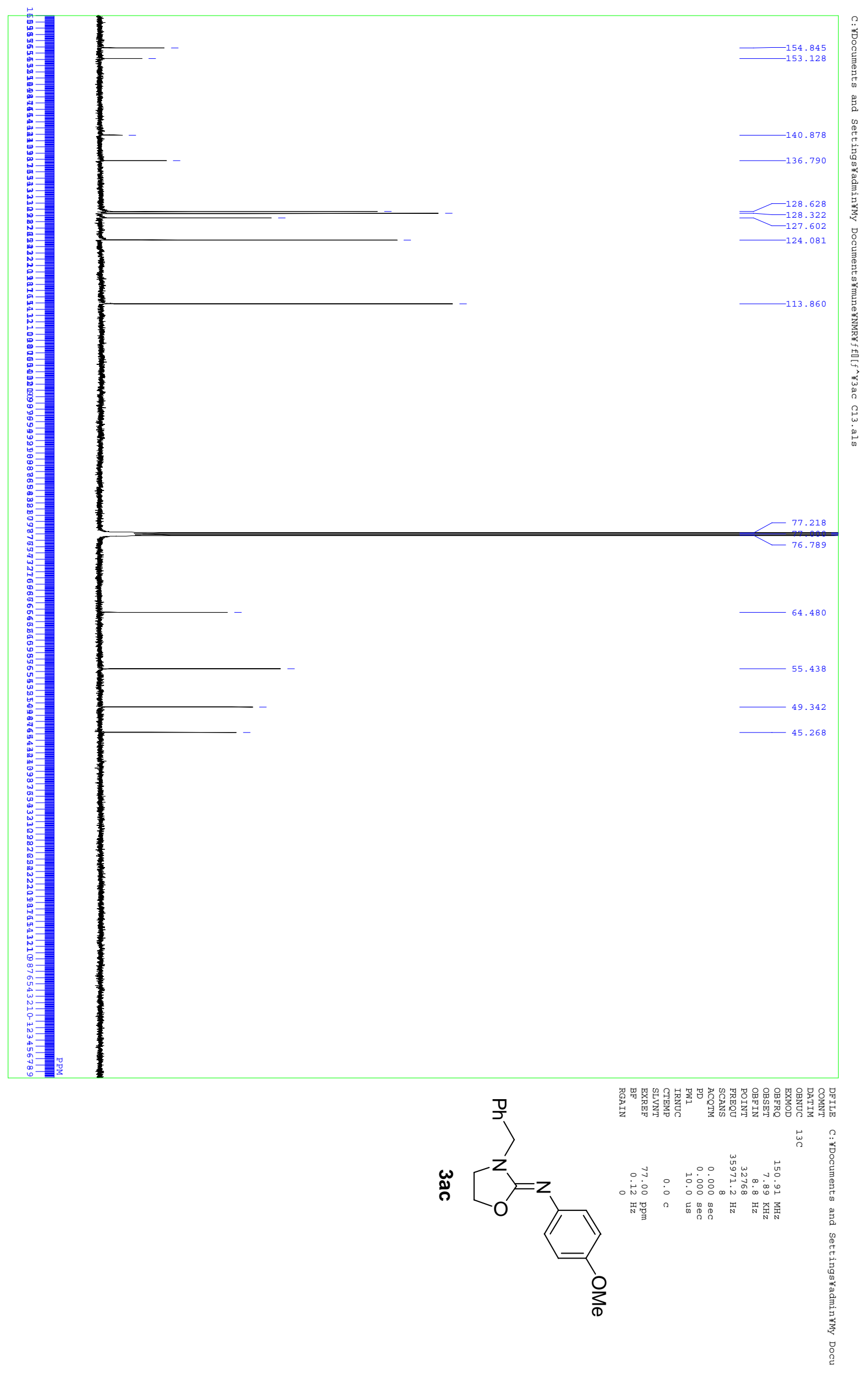




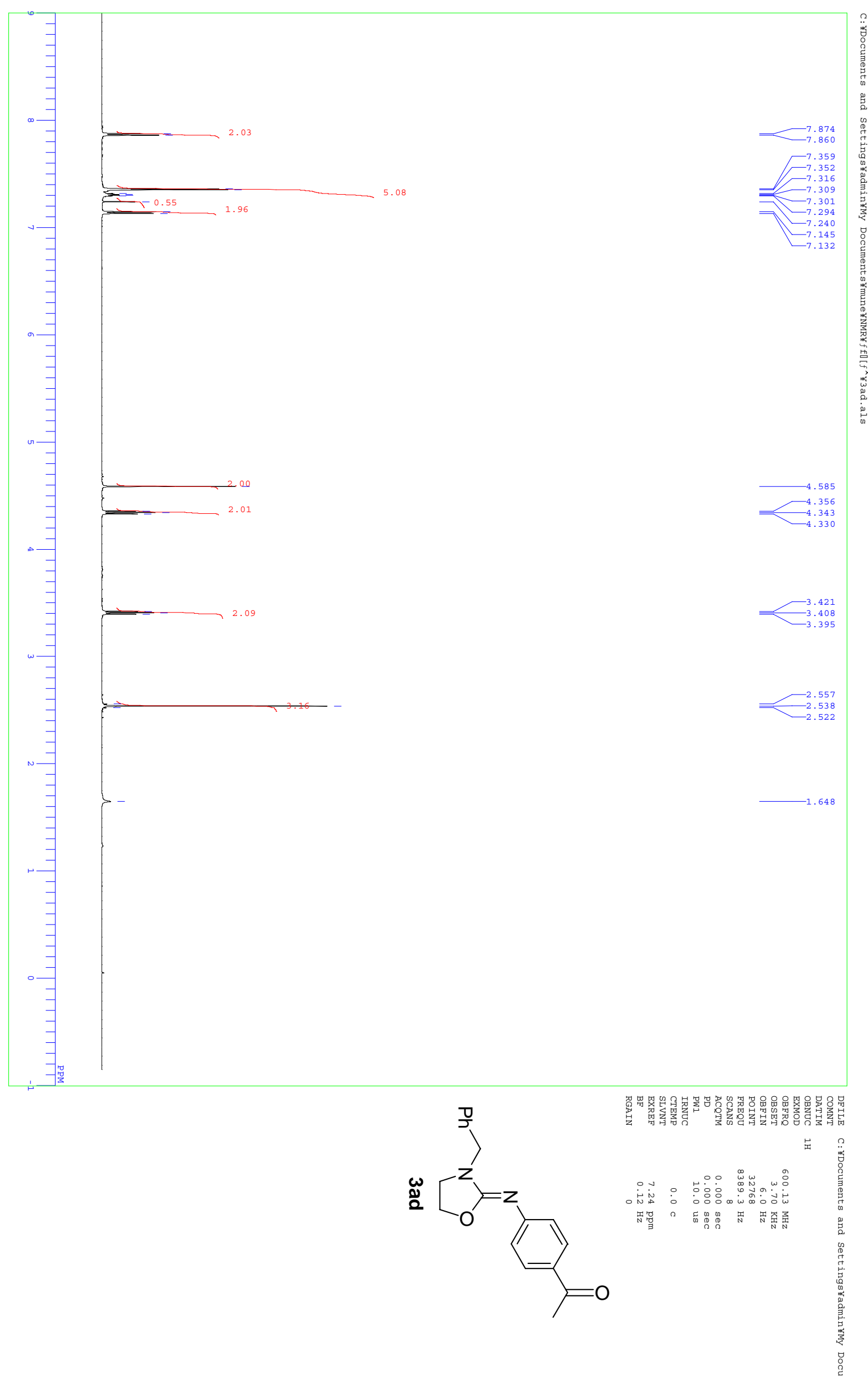




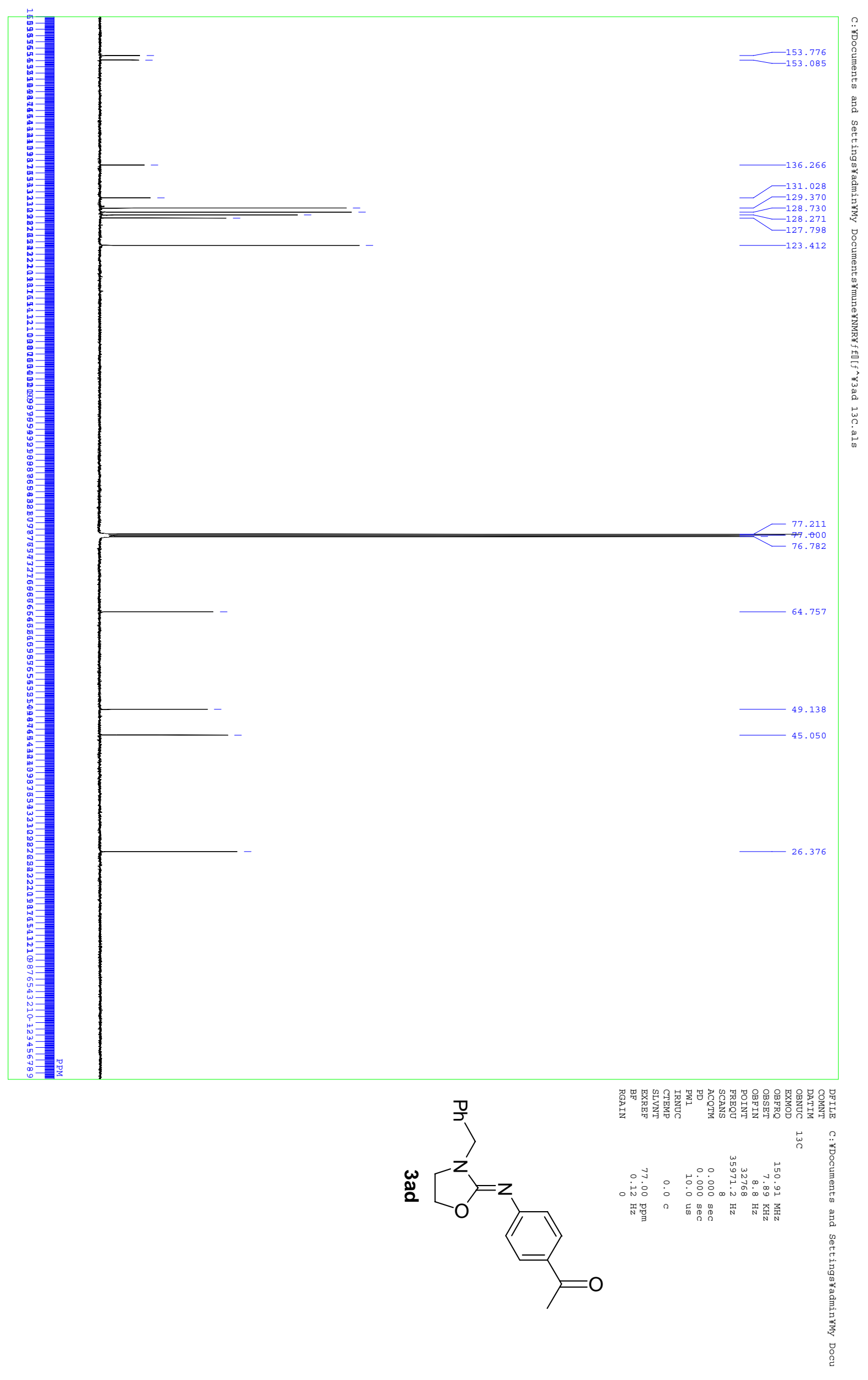




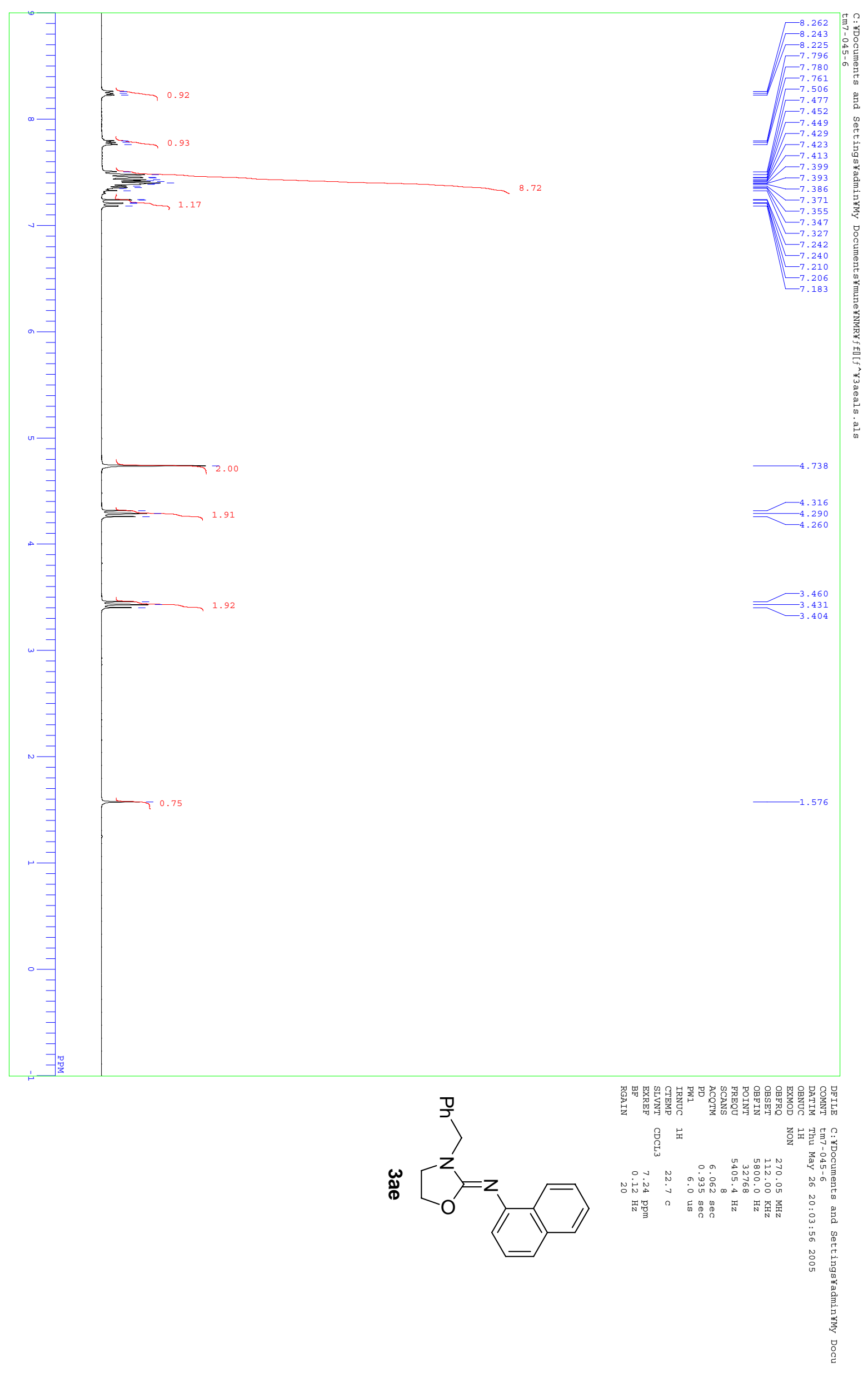



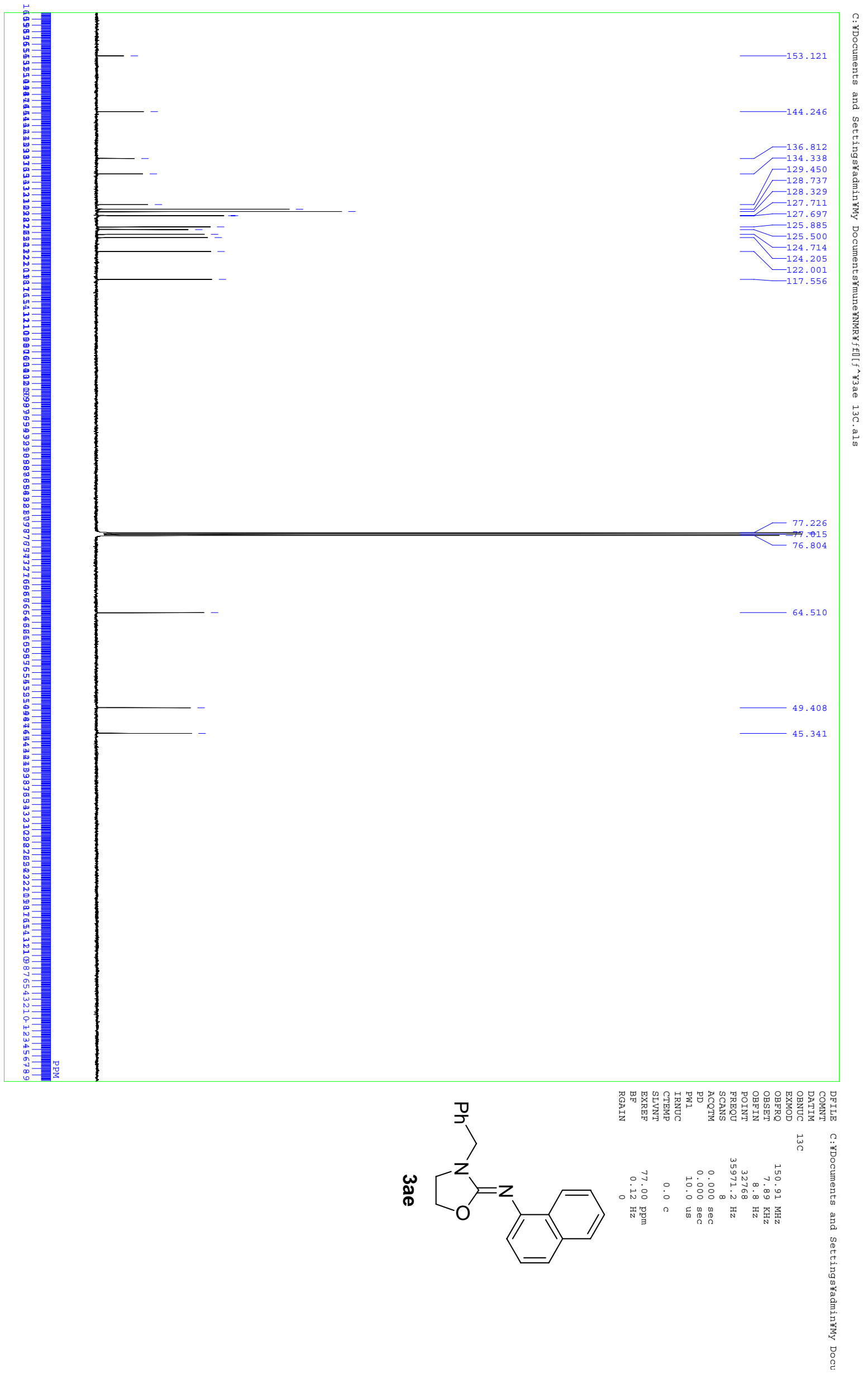